\title{
Composition of the galactic center star cluster
}

\section{Population analysis from adaptive optics narrow band spectral energy distributions ${ }^{\star}$}

\author{
R. M. Buchholz ${ }^{1}$, R. Schödel ${ }^{2,1}$, and A. Eckart ${ }^{1,3}$ \\ 1 I. Physikalisches Institut, Universität zu Köln, Zülpicher Str. 77, 50937 Köln, Germany \\ e-mail: [buchholz; eckart]@ph1.uni-koeln.de \\ 2 Instituto de Astrofísica de Andalucía (IAA)-CSIC, Camino Bajo de Huétor 50, 18008 Granada, Spain \\ e-mail: rainer@iaa.es \\ 3 Max-Planck-Institut für Radioastronomie, Auf dem Hügel 69, 53121 Bonn, Germany
}

Received 10 December 2008 / Accepted 17 March 2009

\section{ABSTRACT}

\begin{abstract}
Context. The GC is the closest galactic nucleus, offering the unique possibility of studying the population of a dense stellar cluster surrounding an $\mathrm{SMBH}$.

Aims. The goals of this work are to develop a new method of separating early and late type stellar components of a dense stellar cluster based on narrow band filters, applying it to the central parsec of the GC, and conducting a population analysis of this area. Methods. We use AO assisted observations obtained at the ESO VLT in the NIR H-band and 7 intermediate bands covering the NIR $K$-band. A comparison of the resulting SEDs with a blackbody of variable extinction then allows us to determine the presence and strength of a $\mathrm{CO}$ absorption feature to distinguish between early and late type stars.

Results. This new method is suitable for classifying $\mathrm{K}$ giants (and later), as well as B2 main sequence (and earlier) stars that are brighter than $15.5 \mathrm{mag}$ in the $K$ band in the central parsec. Compared to previous spectroscopic investigations that are limited to 13-14 mag, this represents a major improvement in the depth of the observations and reduces the needed observation time. Extremely red objects and foreground sources can also be reliably removed from the sample. Comparison to sources of known classification indicates that the method has an accuracy of better than $\sim 87 \%$. We classify 312 stars as early type candidates out of a sample of 5914 sources. Several results, such as the shape of the KLF and the spatial distribution of both early and late type stars, confirm and extend previous works. The distribution of the early type stars can be fitted with a steep power law $\left(\beta_{1^{\prime \prime}}=-1.49 \pm 0.12\right)$, alternatively with a broken power law, $\beta_{1-10^{\prime \prime}}=-1.08 \pm 0.12, \beta_{10-20^{\prime \prime}}=-3.46 \pm 0.58$, since we find a drop in the early type density at $\sim 10^{\prime \prime}$. We also detect early type candidates outside of $0.5 \mathrm{pc}$ in significant numbers for the first time. The late type density function shows an inversion in the inner $6^{\prime \prime}$, with a power-law slope of $\beta_{R<6 \prime}=0.17 \pm 0.09$. The late type KLF has a power-law slope of $0.30 \pm 0.01$, closely resembling the KLF obtained for the bulge of the Milky Way. The early type KLF has a much flatter slope of $(0.14 \pm 0.02)$. Our results agree best with an in-situ star formation scenario.
\end{abstract}

Key words. Galaxy: center - stars: early-type - stars: late-type - infrared: stars

\section{Introduction}

The Galactic center (GC) contains the densest star cluster in the Galaxy with a $\sim 4.0 \times 10^{6} M_{\odot}$ supermassive black hole at its dynamical center (Eckart et al. 2002; Schödel et al. 2002, 2003; Ghez et al. 2003, 2008; Gillessen et al. 2009). Around the black hole, the projected distribution of the stars can best be described by a broken power law (break radius $R_{\text {break }}=66^{\prime \prime} 0 \pm 11^{\prime \prime} 0$, all values given here are projected radii), with a power-law slope of $\Gamma=0.19 \pm 0.05$ within the break radius and $\Gamma=0.75 \pm$ 0.10 towards the outside of the cluster (Schödel et al. 2007). The Galactic center is located at a distance of $\sim 8.0 \mathrm{kpc}$ (Reid 1993; Eisenhauer et al. 2005; Groenewegen et al. 2008; Ghez et al. 2008; Gillessen et al. 2009) from the sun. We adopt this value throughout this work.

\footnotetext{
* A table of all classified sources (5914) is only available in electronic form at the CDS via anonymous ftp to

cdsarc.u-strasbg.fr $(130.79 .128 .5)$ or via

http://cdsweb.u-strasbg.fr/cgi-bin/qcat?J/A+A/499/483
}

The stellar composition of the cluster depends on the distance to the center. This has been observed first as a drop in $\mathrm{CO}$ absorption strength towards Sgr $\mathrm{A}^{*}$ in seeing-limited observations (Allen et al. 1990; Sellgren et al. 1990; Haller et al. 1996). Two explanations have been discussed for this: a significantly lower density of late type stars in the central few arcseconds and/or the presence of a large number of bright early type stars. Adaptive optics assisted observations with high spatial resolution have shown that there is indeed an increased number of early-type stars in this region, while the relative number of late type stars decreases (Genzel et al. 2003; Eisenhauer et al. 2005; Paumard et al. 2006; Lu et al. 2009). Several authors have tried to explain this finding by collisions between stars (or between stellar mass black holes and stars), which may lead to the destruction of the envelopes of giant stars in the central region (Davies et al. 1991; Bailey \& Davies 1999; Rasio \& Shapiro 1990; Davies et al. 1998; Alexander et al. 1999; Dale et al. 2008).

Several stellar populations have been detected in the central parsec: the oldest observable objects that make up the bulk of the visible sources outside of the innermost few arcseconds are old, 
metal-rich M, K and G type giants with ages of $1-10 \times 10^{9}$ years. The helium-burning red clump sources are also present, although they have not been discussed in detail until recently (Maness et al. 2007), because older works on the stellar population did not reach the necessary lower magnitude limit.

A number of intermediate-bright $\left(\operatorname{mag}_{K} \sim 10-12\right)$ stars that are now on the AGB (Krabbe et al. 1995; Blum et al. 1996, 2003) have been produced by a star formation event $\geq 100 \times 10^{6}$ years ago. These stars can be distinguished from late type giants by the $\mathrm{H}_{2} \mathrm{O}$ absorption bands in their spectra that are detectable even at low spectral resolution. Very few supergiants like IRS 7 are also present in the central parsec.

Several objects with featureless, but very red spectra have also been detected (Becklin et al. 1978; Krabbe et al. 1995; Genzel et al. 1996), namely IRS1W, 3, 9, 10W and 21. With high-resolution imaging, most of these sources have been resolved. They are mostly associated with the mini-spiral, and can be interpreted as young and bright stars with rapid mass loss interacting with the interstellar medium in the GC, so called bowshock sources (Tanner et al. 2002, 2003; Geballe et al. 2004; see also Perger et al. 2008).

In the central $\sim 0.5 \mathrm{pc}$ there exists yet another distinct stellar population: massive, young stars created in a starburst $3-7 \times$ $10^{6}$ years ago (e.g., Krabbe et al. 1995). These stars can be found, e.g., in the IRS 16 and IRS 13 associations (e.g. Eckart et al. 2004; Maillard et al. 2004; Lu et al. 2005). The brightest of those young stars have been described as stars in a transitional phase between $\mathrm{O}$ supergiants and Wolf-Rayet stars (WN9/Ofpe according to e.g. Allen et al. 1990), with high mass-loss during this phase (Najarro et al. 1994; Krabbe et al. 1995; Morris et al. 1996; Najarro et al. 1997; Paumard et al. 2001; Moultaka et al. 2005). These stars account for a large part of the luminosity of the central cluster and also contribute half of the excitation/ionizing luminosity in this region (Rieke et al. 1989; Najarro et al. 1997; Eckart et al. 1999; Paumard et al. 2006; Martins et al. 2007). Recently, Muzic et al. (2008) have identified a co-moving group of highly reddened stars north of IRS 13 that may be even younger objects.

Besides the most massive early type stars, a large number of OB stars with masses of $\sim 10-60 M_{\odot}$ have been examined by Levin \& Beloborodov (2003); Genzel et al. (2003); Paumard et al. (2006); Lu et al. (2009). At least 50\% of the early-type stars in the central $0.5 \mathrm{pc}$ appear to be located within a clockwise (in projection on the sky) rotating disk, which was first detected by Levin \& Beloborodov (2003). Later, Genzel et al. (2003); Paumard et al. (2006) claimed the existence of a second, counter-clockwise rotating disk. A very detailed analysis by Lu et al. (2009), based on the fitting of individual stellar orbits, shows only one disk and a more randomly distributed off-disk population (with the number of stars in the disk similar to that on random orbits). Bartko et al. (2009), on the other hand, claim they at least observe a counter-clockwise structure that could be a strongly warped, possibly dissolving second disk. In the immediate vicinity of $\mathrm{Sgr} \mathrm{A}^{*}$, there is yet another distinct group of stars, which form a small cluster of what appear to be early B-type stars (Eckart et al. 1999; Ghez et al. 2003; Eisenhauer et al. 2005). These so-called "S-stars" stars are on closed orbits around $\mathrm{Sgr} \mathrm{A}^{*}$, with velocities of up to a few thousand $\mathrm{km} \mathrm{s}^{-1}$ and at distances as close as a few lightdays (Schödel et al. 2003; Ghez et al. 2003, 2005; Eisenhauer et al. 2005; Ghez et al. 2008; Gillessen et al. 2009). Their orbits have been used to determine the mass of the black hole and the distance to the GC.

How exactly star formation can take place in the central parsec under the observed conditions is still a debated issue.
Classical star formation from gas of the observed density is severely impeded by the tidal shear exerted by the black hole and the surrounding dense star cluster (Morris et al. 1993). Two scenarios are being discussed to explain the presence of the early type stars: Genzel et al. (2003); Goodman (2003); Levin \& Beloborodov (2003); Milosavljevic \& Loeb (2004); Nayakshin \& Cuadra (2005); Paumard et al. (2006) suggest a model of in-situ star formation, where the infall and cooling of a large interstellar cloud could lead to the formation of a gravitationally unstable disk and the stars would be formed directly out of the fragmenting disk. An alternative scenario has been proposed by Gerhard (2001); McMillan \& Portegies Zwart (2003); Portegies Zwart et al. (2003); Kim \& Morris (2003); Kim et al. (2004); Guerkan \& Rasio (2005) with the infalling cluster scenario, where the actual star formation takes place outside of the hostile environment of the central parsec. Bound, massive clusters of young stars can then be transported towards the center within a few Myr (dynamical friction in a massive enough cluster lets it sink in much more rapidly than individual stars, see Gerhard 2001). Recent data seem to favor continuous, in situ star-formation (e.g. Nayakshin \& Sunyaev 2005; Paumard et al. 2006; Lu et al. 2009).

The existence of the $\mathrm{S}$ stars so close to $\mathrm{Sgr} A *$ is yet another matter, known as the "paradox of youth" (Ghez et al. 2003). Two explanations are discussed for the presence of these stars, though neither is satisfactory: formation out of colliding or interacting giants (Eckart et al. 1993; Genzel et al. 2003; Ghez et al. 2003, 2005) or scattering from the disk of young stars (Alexander \& Livio 2004).

Detailed spectroscopic studies of the stellar population in the central parsec have so far only been conducted in the innermost few arcseconds and on small areas in the outer regions of the cluster (see Ghez et al. 2003, 2008; Eisenhauer et al. 2005; Paumard et al. 2006; Maness et al. 2007). Here the main limitation is that the high surface density of sources in the GC forces the observers to use high spatial resolution observations in order to be able to examine all but the brightest stars. However, the field-of-view of integral field spectrometers is quite small at the required angular resolutions (e.g., between $3^{\prime \prime} \times 3^{\prime \prime}$ and $0.8^{\prime \prime} \times 0.8^{\prime \prime}$ for the ESO SINFONI instrument). The aim of this work is to provide a reliable method that allows the classification of several thousands of stars as late or early type in the central parsec down to a magnitude limit of 15.5. These data will then be used to obtain constraints on the stellar population and examine the distribution of early and late type stars. The distribution of very red objects will also be addressed. For this, information about the spectra of the stars is necessary. In Sect. 2.1, we present our method of intermediate band imaging as well as the photometry and calibration. The disadvantage of this method is the very low spectral resolution (only 7 datapoints over the $K$ band and one $\mathrm{H}$ band point) that makes it impossible to detect any but the broadest spectral features. Also, line-of-sight velocities cannot be measured this way. The big advantage is the possibility to cover a large field of view of $40^{\prime \prime} \times 40^{\prime \prime}$ in a very time efficient way. The data analysis in Sects. $2.2-2.7$ will be followed by a population analysis in Sect. 3. We summarize and discuss the implications of our results in Sect. 4.

\section{Observation and data reduction}

\subsection{Observation}

The observations used here were carried out with the NAOSCONICA (NACO) instrument at the ESO VLT unit telescope 
Table 1. Details of the observations used for this work. $N$ is the number of exposures that were taken with a given detector integration time (DIT). NDIT denotes the number of integrations that were averaged online by the read-out electronics during the observation. The scale of all observed images is $0.027^{\prime \prime}$ per pixel.

\begin{tabular}{cccccc}
\hline \hline Date & $\lambda_{\text {central }}[\mu \mathrm{m}]$ & $\Delta \lambda[\mu \mathrm{m}]$ & $N$ & NDIT & DIT [s] \\
\hline 29 April 2006 & 1.66 & 0.33 & 31 & 28 & 2 \\
09 July 2004 & 2.00 & 0.06 & 8 & 4 & 36 \\
12 June 2004 & 2.06 & 0.06 & 96 & 1 & 30 \\
12 June 2004 & 2.24 & 0.06 & 99 & 1 & 30 \\
09 July 2004 & 2.27 & 0.06 & 8 & 4 & 36 \\
09 July 2004 & 2.30 & 0.06 & 8 & 4 & 36 \\
12 June 2004 & 2.33 & 0.06 & 99 & 1 & 30 \\
09 July 2004 & 2.36 & 0.06 & 8 & 4 & 36 \\
\hline
\end{tabular}

4 on Paranal in June/July 2004 and April 2006 (programs 073.B-0084(A), 073.B-0745(A), 077.B-0014(A)). We used an $H$ band broadband filter and seven intermediate band filters (see Table 1).

The seeing varied for the different observations, within a range of 0.5 to $1.3^{\prime \prime}$. The bright supergiant IRS 7 located about 6 " north of Sgr A* was used as the guide star for the adaptive optics (AO) correction, using the infrared wavefront sensor. The sky background was sampled by taking several dithered exposures of a dark cloud near the GC, 713" west and 400" north of Sgr A*, a region largely devoid of stars. A rectangular dither pattern was used for most observations, while some were randomly dithered. All images were flatfielded, sky subtracted and corrected for dead/hot pixels.

In order to be able to separate early and late type sources using the method described in Sect. 2.7, the photometry has to be accurate enough to clearly identify the feature used for the classification (see Sect. 2.4). This means that the typical photometric error should be much lower than the typical depth of the classification feature (see Sect. 2.4 for an estimate of the required accuracy). When observing a large field of view like in this case, a good AO correction can only be achieved within the isoplanatic patch, a region of $\sim 10-15^{\prime \prime}$ for our dataset. This effect leads to a decrease of the Strehl ratio towards the outer regions of the field (see Fig. 1). The values shown in this figure were computed with the strehl algorithm in the ESO eclipse software package $^{1}$ from the PSFs determined in $12 \times 12$ subimages of the IB227 image (with the same PSF stars that were used in the photometry). The parameters of the telescope (like the aperture) were also taken into account. The Strehl values exhibit a clear trend towards lower values at larger projected distances from the guide star. Sources outside of the isoplanatic patch are elongated towards IRS 7. This is a problem when using PSF fitting photometry, while aperture photometry (which is less dependent on the shape of the PSF) is faced with the problem of crowding.

\subsection{Photometry}

In order to counter the aforementioned problems and to achieve reliable relative photometry over the entire FOV which is considerably larger than the isoplanatic patch, a two-step deconvolution process was used. Deconvolution is a good way to reduce source confusion in a crowded field, but it can only be applied satisfactorily if the PSF is very well known and uniform over the

\footnotetext{
1 See N. Devillard, "The eclipse software", The messenger No. 87 March 1997, publicly available at http://www.eso.org/projects/ aot/eclipse/distrib/index.html
}

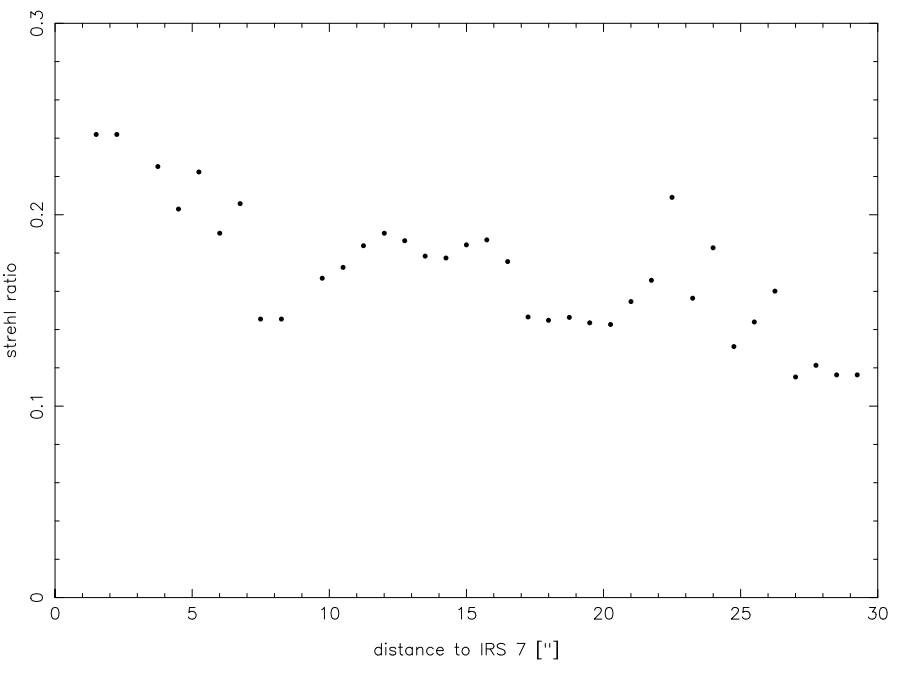

Fig. 1. The strehl ratio, a value that measures the deviation of the PSFs of the sources from an ideal PSF, decreases with the distance to the guide star.

whole field. This process is described in detail in Schödel et al. (in preparation), who are using the same photometry.

The individual lists of detected stars in the filters were merged to a common list of stars detected in all 8 filters. The number of common sources was limited by the image with the lowest quality to 5914. This number includes almost all brighter sources $\left(\operatorname{mag}_{K \mathrm{~s}} \leq 16\right)$, but several extended sources (like the bowshock sources in the northern arm of the minispiral) were not detected in all filters with sufficiently low photometric and position uncertainties.

\subsection{Primary calibration}

The primary calibration done here served a dual purpose: on the one hand, the measured counts for each source in each band were converted into a magnitude (absolute calibration). On the other hand, the bands had to be calibrated relative to each other to ensure smooth spectral energy distributions (SEDs) as they can be expected at this spectral resolution (see Fig. 3). Only very broad spectral features like $\mathrm{CO}$ bandheads and $\mathrm{H}_{2} \mathrm{O}$ absorption bands are expected to be observable here. Even the feature around $2.06 \mu \mathrm{m}$ that appears in the B0V spectrum only causes a decrease of $0.014 \mathrm{mag}$ in that filter compared to the K4.5III spectrum that lacks this feature. This can be neglected compared to other photometric uncertainties.

Atmospheric features can also influence the shape of the measured SEDs and need to be eliminated. But the most important parameter that controls the quality of the data turned out to be the AO performance, which is why we did not use two of the available intermediate band datasets $(2.12$ and $2.18 \mu \mathrm{m})$.

These goals were achieved by calibrating the common list of sources with 11 known OB stars (see Table 2 and Fig. 2) close to Sgr A*. We adopted the classifications of Paumard et al. (2006), who provided a list of 90 early type stars in the central parsec. Stars of this type have some emission lines in the $K$ band (see Fig. 3 upper right), e.g. the HeI line at $2.058 \mu \mathrm{m}$, but these lines are narrow enough to be negligible compared to the continuum at the spectral resolution of our data. Thus, the spectra of these stars can be assumed to be featureless, so they can be described by a blackbody spectrum with an effective temperature of $\sim 30000 \mathrm{~K}$. The final calibration has only a minimal dependance on the assumed effective temperature because the 
Table 2. Early type calibration stars, used for the primary calibration (names, types and $K$ band magnitudes according to Paumard et al. (2006), approximate values for $T_{\mathrm{eff}}$ from Allen's Astrophysical Quantities).

\begin{tabular}{lllll}
\hline \hline Name & ID & mag $K$ & Type & $T_{\text {eff }}$ \\
\hline E69 & 61 & 11.32 & early & \\
E55 & 195 & 12.45 & B0-1I & $\sim 20000$ \\
E47 & 219 & 12.50 & B0-3I & $\sim 18000$ \\
IRS 16SSE1 & 148 & 11.90 & O8.5-9.5I & $\sim 32000$ \\
IRS 16SSE2 & 199 & 12.10 & B0-0.5I & $\sim 20000$ \\
IRS 16SSW & 71 & 11.45 & O8-9.5I & $\sim 32000$ \\
IRS 33N & 58 & 11.22 & B0.5-1I & $\sim 20000$ \\
E22/W10 & 220 & 12.73 & O8-9.5I/III & $\sim 32000$ \\
E25/W14 & 215 & 12.58 & O8.5-9.5I & $\sim 32000$ \\
E43 & 126 & 12.10 & O8.5-9.5I & $\sim 32000$ \\
E53 & 254 & 12.31 & B0-1I & $\sim 20000$ \\
\hline
\end{tabular}

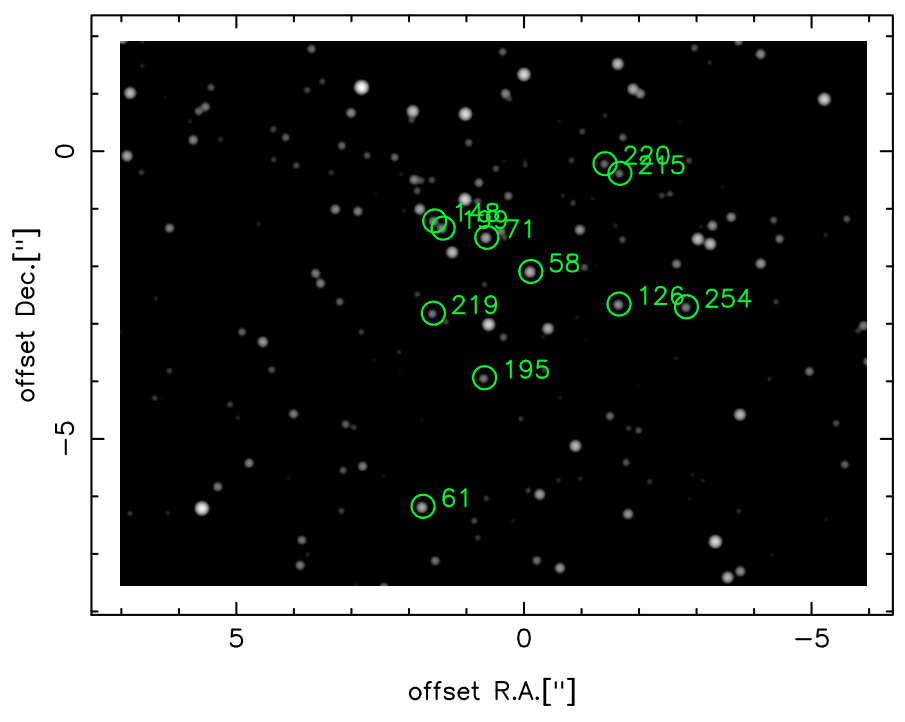

Fig. 2. Sources used for the primary calibration. Numbers correspond to the numbers in the common list, as also shown in Table 2.

Rayleigh-Jeans law is a very good approximation for the SED of hot stars in the near-infrared (using $20000 \mathrm{~K}$ instead leads to a difference of only $0.01 \mathrm{mag}$ in the reference magnitudes). Therefore, the same $T_{\text {eff }}$ can be used for all calibration sources.

Extinction towards the GC is significant even in the nearinfrared. An extinction of $\sim 3.0 \mathrm{mag}$ (Scoville et al. 2003; Schödel et al., in preparation) has been measured towards the central parsec in the $K$ band. In order to minimize calibration uncertainties, we used individual extinction values for each calibration source, taken from the extinction map provided by Schödel et al. (in preparation). Since the extinction also depends on the wavelength, we used the Draine (1989) extinction law to calculate an extinction value for each band from the basic value for the $K$ band, i.e. we assumed:

$A_{\lambda} \propto \lambda^{-1.75}$.

Using an extinction law with a different exponent (like $\lambda^{-2.0}$ as proposed by Nishiyama et al. 2008) would lead to a general offset of about $0.5 \mathrm{mag}$ in extinction, but neither the relative distribution of the extinction values nor the results of our classification would vary, since the same extinction law is used again to fit for the individual extinction of each source in the classification process.
The continuous extincted blackbody spectra were converted to 8 point SEDs by multiplying them with the transmission curve of each filter (see Fig. 3, lower left). By comparing these theoretical SEDs with the measured counts of the calibration stars, a calibration factor was calculated for each filter to convert the observed counts of each star into a magnitude. This eliminates the influence of any atmospheric features, since they should occur in all sources and thus also in the calibration sources. The magnitude at $2.24 \mu \mathrm{m}$ was adopted as the $K \mathrm{~s}$ band magnitude of the source, since this band is the closest available one to the center of the $K \mathrm{~s}$ band and not affected by absorption features. For the classification algorithm and the analysis, we used extinction corrected magnitudes, i.e. we modified the measured magnitudes with the difference between the individual extinction of each source and the average extinction. This step eliminates the effect that the variable extinction in the observed region has on the brightness of the sources, which is necessary since the cutoff used for the classification depends on the brightness of the source in question. This step also eliminates the effects of spatially variable reddening on the derived luminosity functions. We assumed an average extinction in the central parsec of 3.3 mag (Schödel et al. in preparation).

$\operatorname{mag}_{\mathrm{ext}}=\operatorname{mag}_{K \mathrm{~s}}-A_{K \mathrm{~s}}+A_{\mathrm{avg}}$

here, $A_{K \mathrm{~s}}$ denotes the measured individual extinction of each source in magnitudes (in the $K \mathrm{~s}$ band), $A_{\text {avg }}=3.3 \mathrm{mag}$ is the average $K \mathrm{~s}$ band extinction in the central parsec, $\operatorname{mag}_{K \mathrm{~s}}$ represents the measured Ks band magnitude at $2.24 \mu \mathrm{m}$.

After the primary calibration, the sources within a few arcseconds of Sgr A* show SEDs that agree very well with SEDs calculated from template spectra of typical stars (Wallace \& Hinkle 1997). The optimum of the calibration is centered around the region where the primary calibration sources are located (close to Sgr A*), and not around the guide star. With increasing distance from the optimum, the SEDs appear to show systematic deviations correlated over areas of a few arcseconds size. There are unfortunately not nearly enough known early type stars over the whole field to extend our primary calibration to the whole central parsec, so an additional local calibration had to be introduced that makes use of another class of stars that are easy to identify and abundant over the whole field: horizontal branch/red clump (HB/RC) stars. We assume that almost all late type stars with $K$ band magnitudes between 14.5 and 15.5 are part of this population. This leaves the problem of telling apart late and early type stars. The criterion for this is the same that is later used in the final classification: the CO band depth (CBD).

\subsection{CO band depth as a classification feature}

Since only very broad spectral features are visible at the low spectral resolution of our data, it is not possible to determine the exact spectral type of every single source. The feature used to distinguish between early and late types is the region beyond $2.24 \mu \mathrm{m}$, where late type stars show characteristic CO band head absorption. At the spectral resolution available here, no individual lines or band heads are visible, but a broad feature like the $\mathrm{CO}$ absorption alters the shape of the SED in the corresponding region significantly (see Fig. 4). The presence of $\mathrm{CO}$ band heads causes a significant dip in the SED for wavelengths greater than $2.24 \mu \mathrm{m}$. In general, the presence of this feature leads to the classification of the source as a late type star with our method, while its absence makes the star an early type candidate. We did not consider young stellar objects with $\mathrm{CO}$ band head emission in 

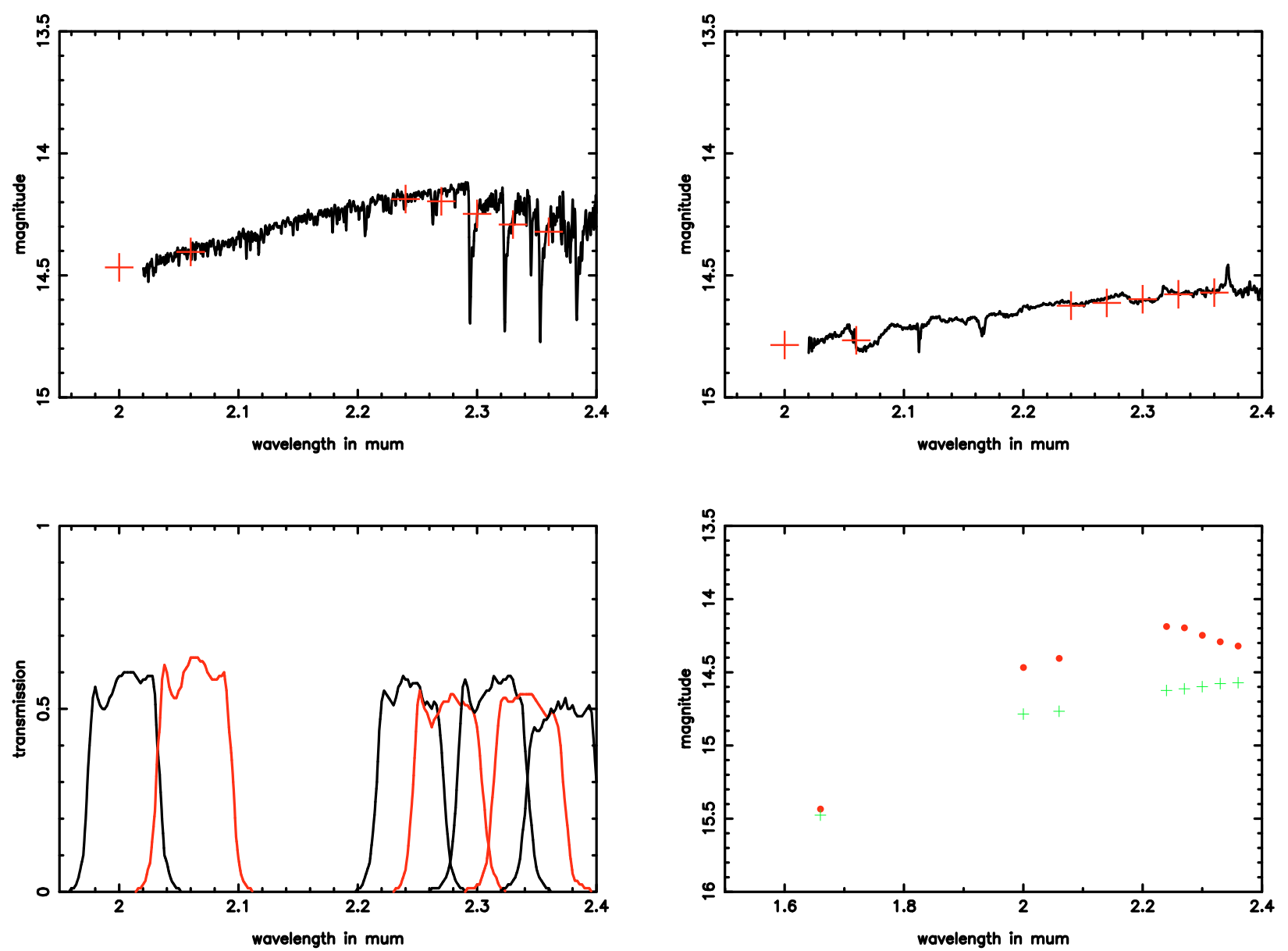

Fig. 3. Conversion of continuous spectra into template SEDs. Upper left: K4.5 giant spectrum, the crosses indicate the low resolution SED, $A_{K}=3.3$ mag. Upper right: B0 main sequence spectrum, $A_{K}=3.3$ mag. Lower left: transmission curves of IB filters. Lower right: K4.5III and BOV template SEDs with added $H$ band datapoint calculated from effective temperature and extinction.

our analysis. If any such objects exist within our data, they were treated as early-type stars.

This feature also sets the limit for the required photometric accuracy: if a late type source with a $K$ s band magnitude of 15.5 is expected to show a $\mathrm{CO}$ bandhead feature with a depth of $0.1 \mathrm{mag}$, this corresponds to a difference of $\sim 11 \%$ in flux at $2.36 \mu \mathrm{m}$ and less at shorter wavelengths. Thus, the photometric error should be significantly lower than that value, which is achieved by our method for most sources. If the error of a single data-point exceeded $15 \%$, the source was excluded completely.

We determined the presence of this feature in the following way: an extincted blackbody $\left(T_{\text {eff }}=5000 \mathrm{~K}\right.$, but see Sect. 2.7 for the effective temperatures used in the final iteration of the fitting process) was fitted to the first 5 data-points $(\lambda \leq 2.27 \mu \mathrm{m})$. The extinction was varied in a range of 0 to $8 \mathrm{mag}$ in $0.1 \mathrm{mag}$ steps. The best fit was chosen based on the reduced $\chi^{2}$ of the fit. A third order polynomial was then fitted to the complete SED, with the first 5 data-points replaced by the fitted extincted blackbody to ensure a smooth fit. Although it might appear that using only the highest quality filters that are not influenced by possible additional spectral features (like the $H$ band and the 2.24 $\mu \mathrm{m}$ filter) would produce the best results, this would in fact be less reliable than the method we used here. Since the GC is a very crowded stellar field, variable AO performance and related variations of the Strehl ratio can cause variations of the measured fluxes of stars. This can easily lead to outliers in the data. Therefore, it is safer to use a larger number of measurements instead of just two or three filters (see Sect. 2.7 for the special case of AGB stars with intrinsic $\mathrm{H}_{2} \mathrm{O}$ absorption features).

For future applications of this method, we would prefer to also use the intermediate band filter centered at $2.12 \mu \mathrm{m}$. The data available at this wavelength had a low Strehl ratio and thus low photometric accuracy. Using the $2.18 \mu \mathrm{m}$ filter is not advisable, since the data would be influenced by the strong $\mathrm{Br} \gamma$ emission of the minispiral (e.g. Paumard et al. 2004). It would also be possible to make use of the available narrow band filters to probe regions of special interest in the spectra, e.g. Br $\gamma$ emission or absorption features in early type stars or the $2.20 \mu \mathrm{m}$ $\mathrm{NaI}$ absorption feature in late type stars. But for that, a very careful background subtraction would be necessary to eliminate the strong influence of the minispiral (see e.g. Eisenhauer et al. 2005; Paumard et al. 2006, the same problem occurs with spectroscopy). Most important would be achieving improved photometric stability and deeper integration.

The shape of both the fitted extincted blackbody and the third order polynomial depend on depend on the local extinction, but the difference between the extrapolated values of the two fits at $2.36 \mu \mathrm{m}$ is only extremely weekly dependent on extinction. We tested this by artificially reddening a typical late type SED (modeling extinction values of 1.6-6.5 mag) and applying the fitting algorithm to it. The resulting difference of the polynomial and the blackbody varied by less than $0.15 \%$, with slightly higher values for lower extinction. Thus, we can assume that this value is independent of the local extinction within an 

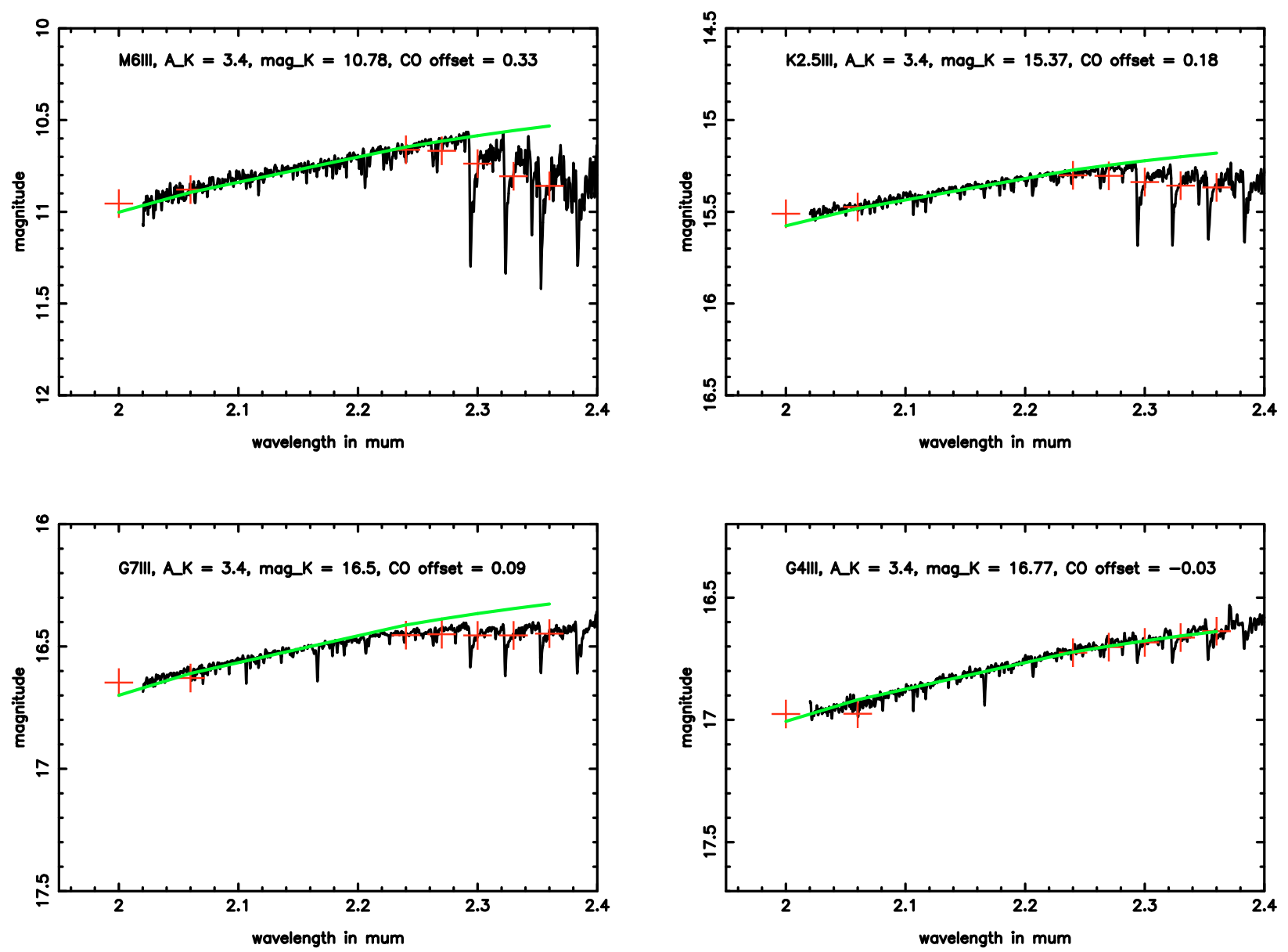

Fig. 4. $K$ band spectra of late type stars expected in the GC. The crosses mark the corresponding SEDs and the green solid line a fitted extincted blackbody. As can be seen, the CO band heads of G giants are not deep and wide enough to have much influence on the low-resolution SEDs.

acceptable margin of error, which means it can be used as a good measure for the presence and depth of $\mathrm{CO}$ band heads and thus as a classification criterion. But in order to separate early and late type stars based on this value, which we will term $C O$ band depth $(C B D)$ in the following, a reliable cutoff is needed.

\subsection{Cutoff determination}

Although one could expect from template SEDs as shown in Fig. 3 a CBD close to zero for early type sources and greater than zero for late type sources, which could be separated by a simple general cutoff, the limited signal-to-noise ratio of the data, combined with additional sources of uncertainty, such as photometric errors due to source crowding (see e.g. Ghez et al. 2008) led to considerable scatter in the CBD feature. Therefore, the CBD value of early type stars scatter to positive and negative values. But still, this value can be used as a useful tool to reliably separate early- and late-type stars statistically.

In order to compensate for these difficulties, we compared the CBD values of the sources classified by Paumard et al. (2006); Maness et al. (2007) that are also present in our dataset. As can be seen in Fig. 5, the early- and late-type sources mostly fall into separate regions when we plot the stellar magnitude vs. the CBD value. However, there are some (less than 5\%) latetype sources present in the early-type region and vice versa. This makes it impossible to use a single smooth cutoff line, let alone a single cutoff value for the whole magnitude range.

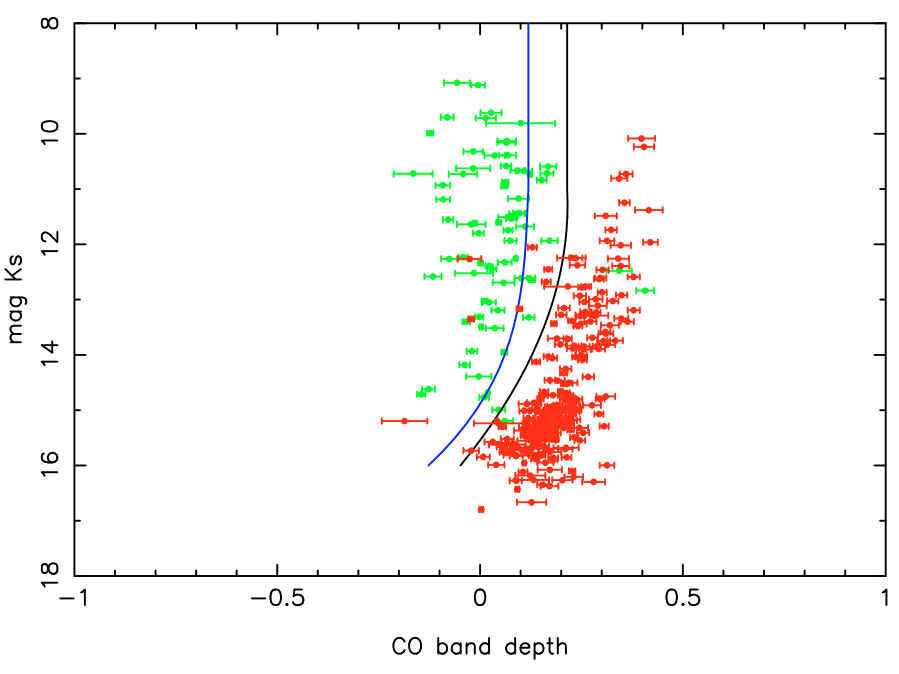

Fig. 5. CO band depth (CBD) at $2.36 \mu \mathrm{m}$ for known early type sources found in this sample (Paumard et al. 2006, green) and known late type sources (Maness et al. 2007, red), with the cutoff lines plotted in black (derived from early type sources for inner 12") and blue (based on late type sources, used outside of $12^{\prime \prime}$ ).

Almost all spectroscopically identified early-type sources are concentrated in the innermost 12.9". Therefore, two different smooth cutoff lines were calculated: one that reliably separates all known early and late type sources in the inner $0.5 \mathrm{pc}$, and 


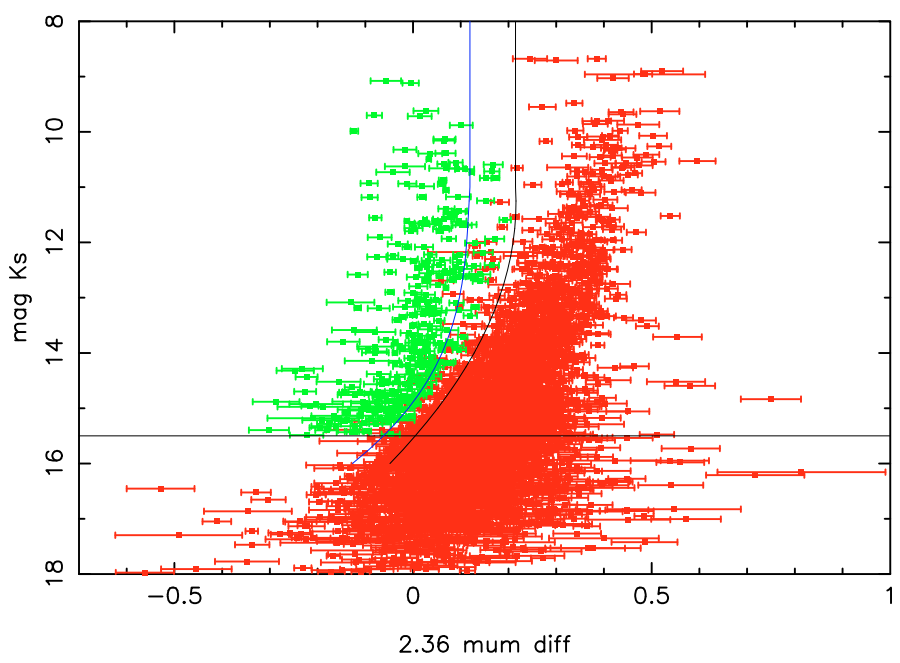

Fig. 6. $\mathrm{CO}$ band depth at $2.36 \mu \mathrm{m}$ for the sources in the central parsec of the GC, with identified early type (green) and late type (red) sources, separated by the inner (black) and outer cutoff (blue solid line).

one for the outer regions that encompassed the known late type sources there (see Fig. 5). This ensures a more reliable detection of early type stars in the outer region (where the early type density is at best very low). The cutoff line based on the early-type stars will underestimate the number of early type stars, just as using the less strict cutoff based on the late-type stars might overestimate the number of early type stars. Use of both cutoff lines allows us to estimate the uncertainty of this method.

We were able to achieve a clear separation of the reference sources with this two-cutoff-method, except a few outliers and noisy sources (see Sect. 3.2).

The theoretical lower magnitude limit of this method is determined by the presence of deep enough $\mathrm{CO}$ band heads so that the CBD significantly exceeds the photometric uncertainties. This was determined from the Wallace \& Hinkle (1997) spectra to be the case for $\mathrm{M}, \mathrm{K}$ and brighter $\mathrm{G}$ giants, corresponding to $\sim 15.5-16 \mathrm{mag}$ at the distance and extinction towards the GC. Fainter G giants and early type stars fainter than B2 have almost identical CBD values (see Fig. 4). The comparison with published spectroscopic identifications and the shape of the CBD vs. magnitude plot (Fig. 6) justified the adoption of a magnitude limit of 15.5 .

\subsection{Local calibration}

With a classification criterion and a reliable cutoff in place, we were able to apply a local calibration that significantly improved the results.

Previously published $K$ band luminosity functions of the central parsec (e.g. Genzel et al. 2003; Maness et al. 2007; Schödel et al. 2007) have shown that the magnitude range of 14.5 to 15.5 is dominated by $\mathrm{HB} / \mathrm{RC}$ stars outside of the innermost arcsecond. In addition, the density of early type stars decreases steeply outside of the innermost arcsecond (Genzel et al. 2003; Paumard et al. 2006; Lu et al. 2009). So even without knowing the exact type of every source in this magnitude range, they can be used as calibration sources assuming a typical late type SED. This template was determined as the average SED of 14 manually selected late type reference stars (see Table 4) close to the optimum of the calibration. These stars were selected based on the similarity of their SEDs to the expected shape.
After this, the $\mathrm{HB} / \mathrm{RC}$ sources in the whole field except the innermost $2^{\prime \prime}$ were selected based on two criteria: an extinction-corrected magnitude between 14.5 and 15.5 and a CBD value above the cutoff. The SEDs of these sources were compared to the template HB/RC SED corrected for the determined individual extinction value. This yielded a calibration factor for each source in each band. Due to the uniform distribution of HB/RC sources over the field, these values map the local deviations and can be used to calibrate the SEDs of all sources. The calibration factors were checked for outliers first. If a calibration factor exceeded the median over the closest 20 sources by more than $3 \sigma$, it was replaced by that median. On average, 20 sources are contained within an area of $\sim 2 \times 2^{\prime \prime}$, depending on the position in the field. This already leads to a spatial resolution of $\sim 2^{\prime \prime}$. The factors were then processed into 8 calibration maps by smoothing the raw maps with a Gaussian with a $2^{\prime \prime}$ FWHM (see Fig. 8).

The final cutoff to be used in the actual classification procedure was calculated in the same way as described in Sect. 2.5, but with the locally calibrated values for the reference sources. This turned out to be a minor adjustment, so the local calibration process did not have to be reiterated.

This local calibration eliminates most systematic local deviations and allows a more reliable classification of the sources towards the outer edges of the field (see Fig. 7).

\subsection{Source classification}

The fitting process described in Sect. 2.4 was repeated on the calibrated data. The extinction, CBD, the $\chi^{2}$ of the fitted polynomial and the uncertainty of the CBD were calculated for each source in several iterations (see below). The reduced $\chi^{2}$ value for the last five data-points of the polynomial fit was used as a criterion for the quality of the fit. Using a reduced $\chi^{2}$ for all eight data-points makes less sense than the method used here, since, on the one hand, the first five data-points are replaced by the fitted blackbody for the polynomial fitting, and on the other hand, the first data-points do not have a large influence on the classification anyway. In order to allow a realistic comparison to the cutoff for the final classification, the uncertainty of the CBD was calculated as the average root-mean-square deviation of the last five data-points to the polynomial fit. This value deliberately ignores the individual photometric uncertainties of the data-points, because sources with noisy SEDs with large uncertainties for the last data-points that are crucial for the classification would otherwise have a similar CBD uncertainty as sources with smooth SEDs with the last data-points close to the fitted blackbody. This could lead to erroneous classifications and is not desirable.

We separated our sample of 5914 stars into the following classes:

1. Noisy sources: if the reduced $\chi^{2}$ of a source was higher than 1.5 , we rated this source as too noisy for classification (334 sources). The cutoff of 1.5 was chosen because it excludes the $5 \%$ most noisy sources.

2. Foreground sources: these stars are not part of the population of the central parsec and have to be removed prior to any analysis. They are easily recognizable by their low fitted extinction value. Every source with a fitted extinction of less than 2 mag was rated as a foreground source here and excluded. 58 sources were classified as foreground sources.

3. Very red objects: several very strongly reddened objects (like e.g. IRS 3) can be observed in the field. Their SEDs are influenced by other effects in addition to their intrinsic stellar 

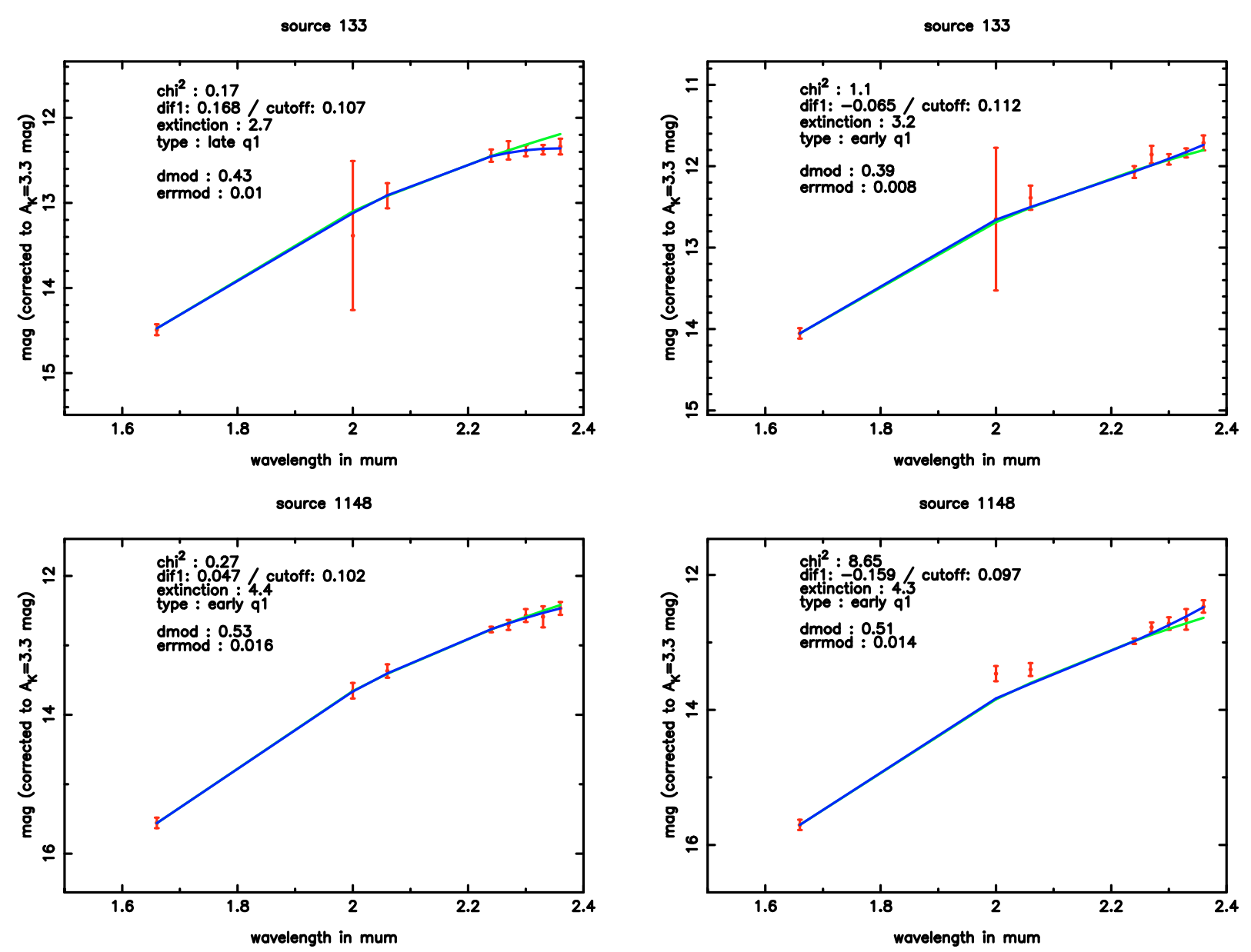

Fig. 7. Two examples for the effects of the local calibration. Upper right: source fitted as early type, but the SED is noisy which is reflected in the $\chi^{2}$. Upper left: same source after local calibration, now fitted as late type, with a much better fit. Lower right: source fitted as early type, noisy SED, several data-points do not agree with the fit, large $\chi^{2}$. Lower left: same source, still fitted as early type, but with much better fit.

features, e.g. dust shells and bow-shocks. These sources were also excluded from the further analysis since they cannot be compared easily to normal early or late type stars. We rated every source with a fitted extinction of more than 5 mag as such an object (24 sources). The high fitted extinction here is just a selection criterion that results from our algorithm. The real extinction toward the sources cannot been determined because of the unknown intrinsic SED of the very red objects.

4. Early type sources (quality 1-3): the CBD of every source brighter than 16 mag (extinction corrected) was compared to the applicable cutoff: sources closer than 11.9" were compared to the inner cutoff, while any source further away than $11.9^{\prime \prime}$ was compared to the outer cutoff. Sources with a CBD below the cutoff were rated as preliminary early type candidates and fitted again with $T_{\text {eff }}=30000 \mathrm{~K}$ which comes much closer to the actual effective temperatures of early type stars than the $5000 \mathrm{~K}$ that were used in the initial fit. A $30000 \mathrm{~K}$ blackbody has a flatter slope in this wavelength regime than a $5000 \mathrm{~K}$ blackbody, so the fitted extinction and the CBD that results from the fit also differ. The updated CBD value was compared to the cutoff again. Only sources brighter than 15.5 mag (extinction corrected) were considered. The extinction fitted in this iteration exceeded the previous value by up to $\sim 0.5$ due to the flatter slope of the blackbody. This explains the higher magnitude cutoff used in the first step. A classification of fainter sources is not possible with this method, because the depth of the CO band head feature becomes too shallow (Wallace \& Hinkle 1997). If the CBD was more than $3 \sigma$ lower than the cutoff and the SED was not too noisy (reduced $\chi^{2}<1.5$ ), the source was rated as an early type with quality 1 (highest quality, 277 sources). If CBD was between 2 and $3 \sigma$ lower than the cutoff, the source was rated as an early type with quality 2 (25 sources). Sources with a CBD $1-2 \sigma$ below the cutoff were rated as early type quality 3 (10 sources).

5. Late type sources (quality 1-2): all other sources that did not meet the cutoff criterion to qualify as an early type candidate were fitted again with $T_{\text {eff }}=4000 \mathrm{~K}$. This value is typical for the expected late type giants. This again resulted in different extinction and CBD values. The uncertainties of the CBD and the reduced $\chi^{2}$ of the fit were calculated in the same way as for the early type stars. If the CBD exceeded the cutoff by more than $1 \sigma$ and the magnitude of the source was brighter than 15.5 , the source was rated as a late type star, quality 1 (2955 sources), the others were rated as late type, quality 2 (2231 sources). The late type quality 2 sources are neglected in the analysis of the late type population, since our method does not provide a clear identification any more.

6. AGB stars: AGB stars are among the brightest sources in the field. Due to their prominent $\mathrm{H}_{2} \mathrm{O}$ absorption feature, they are usually fitted with a too flat blackbody/polynomial: the absorption feature leads to a higher magnitude at 2.00 and $2.06 \mu \mathrm{m}$ that drags the fitted blackbody and the 

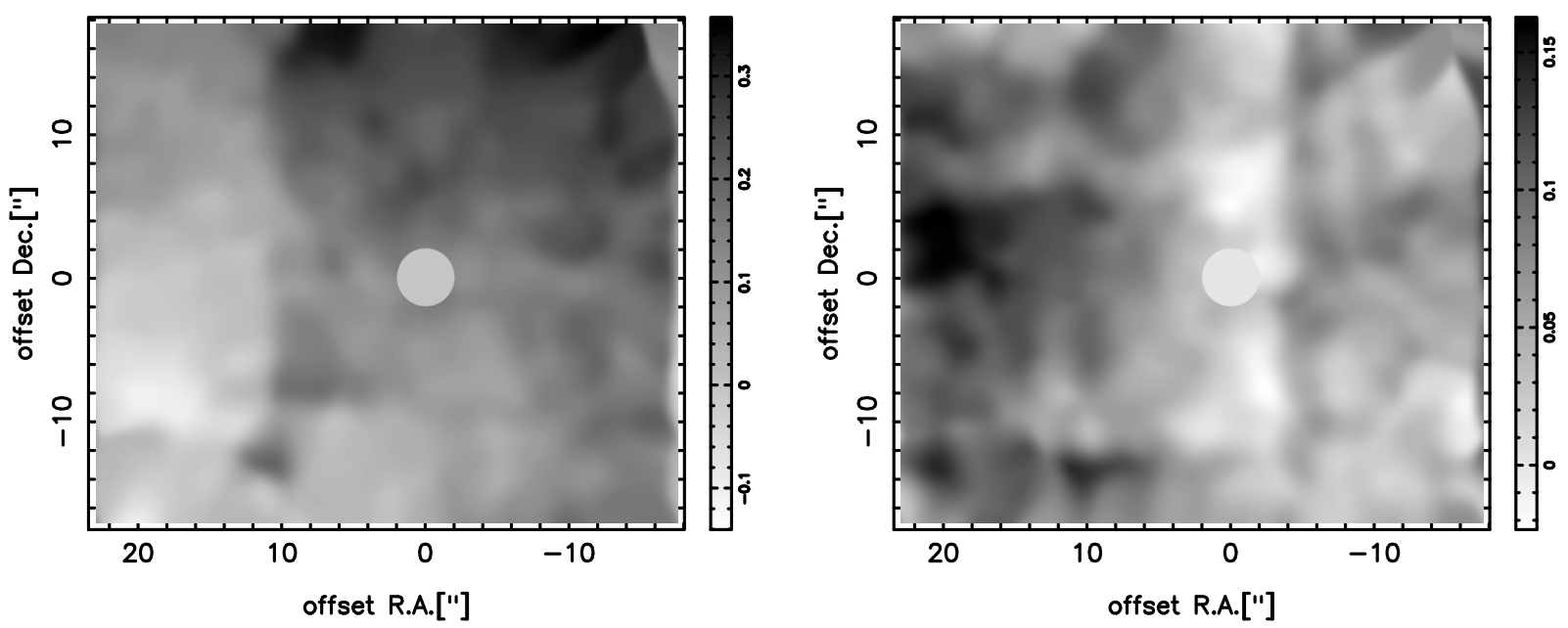

Fig. 8. Examples of the calibration maps used for local calibration (see Sect. 2.6) in the 8 bands. Left: $2.00 \mu \mathrm{m}$. Right: $2.33 \mu \mathrm{m}$. A central region with a radius of $2^{\prime \prime}$ was excluded, the calibration factors were set to 0 there. Residuals of the rectangular dither pattern that was applied in the observations can be seen in the calibration maps.

Table 3. Stellar types expected to be observable in the central parsec, considering the distance and the extinction towards the GC. Values taken from Allen's Astrophysical Quantities, the types shown here are the ones presented in that work that fall into the observational limits for the central parsec.

\begin{tabular}{lrrlrr}
\hline \hline Early types & mag $K$ & $T_{\text {eff }}$ & Late types & $T_{\text {eff }}$ & mag $K$ \\
\hline B3Ia & 11.80 & $\sim 16000$ & M6III & $\sim 3200$ & 10.78 \\
O6I & 12.12 & $\sim 36000$ & M4III & $\sim 3400$ & 12.32 \\
O7V & 13.92 & $\sim 36000$ & M2III & 3540 & 12.92 \\
B0V & 14.65 & 30000 & M0III & 3690 & 13.57 \\
B2V & 16.03 & 20900 & K4.5III & $\sim 4100$ & 14.29 \\
B3V & 16.33 & $\sim 19000$ & K2.5III & $\sim 4300$ & 15.37 \\
B7V & 17.51 & $\sim 13000$ & G8.5III & $\sim 4750$ & 16.42 \\
& & & G7III & $\sim 4900$ & 16.50 \\
& & & G5III & 5050 & 16.62 \\
& & & G4III & $\sim 5100$ & 16.77 \\
& & & G0III & $\sim 5200$ & 17.27 \\
\hline
\end{tabular}

polynomial "down". This can lead to a CBD below the cutoff and a classification as an early type star. To counter this effect, all sources were checked whether or not these two data-points were more than $1 \sigma$ below the fitted blackbody. If that was the case, the fitting was repeated without them. When the resulting classification changed, we adopted the updated class as the better result. We did not deem it necessary to put these objects in a separate sub-category, but we see this modification as a way to further improve the classification of early type stars and remove false candidates. Source 1049 is a good example for this effect, especially since it has been identified spectroscopically as a late type source by Maness et al. (2007). This addition to the algorithm resulted in 8 less candidates for early type stars in total, which does not have a big impact on our results as a whole, although it has a noticeable effect on the density distribution in the outer region of the observed area.

Using only two different values for $T_{\text {eff }}$ to fit early and late type candidates turned out to be sufficient, since the variations in the fitted extinction stayed within $\pm 0.1 \mathrm{mag}$ when we used the minimum resp. maximum values for $T_{\text {eff }}$ as shown in Table 3 for the respective classes of objects. We only determined the extinction in steps of $0.1 \mathrm{mag}$ in the first place, so we considered this slight uncertainty acceptable.
Table 4. Stars close to Sgr A* used to create the HB/RC template. All these sources were classified as late type by our algorithm, which is another self-consistency test.

\begin{tabular}{cc}
\hline \hline ID & mag $K$ \\
\hline 3421 & 15.6 \\
3369 & 15.1 \\
2608 & 15.6 \\
2888 & 15.4 \\
3896 & 16.1 \\
3565 & 15.1 \\
4215 & 15.3 \\
3253 & 15.7 \\
2260 & 15.3 \\
2248 & 15.3 \\
1440 & 14.6 \\
2206 & 14.6 \\
4448 & 14.8 \\
2158 & 15.0 \\
\hline
\end{tabular}

\section{Results and discussion}

\subsection{Stellar classification}

In total, 3349 of 5914 sources have been classified as either early type, late type, foreground or very red sources. 2231 of the remaining sources are too faint to allow a reliable classification with our method, although they can be assumed to be mostly late type sources. The SEDs of 334 sources were too noisy and have been excluded by the classification algorithm.

Unless otherwise indicated, all results shown here are based on the sources with an extinction corrected magnitude brighter than 15.5 (see Eq. (2)). Foreground sources have also been excluded (58 in total), since they do not belong to the population of the central parsec. Very red objects ( 24 sources) have also not been included in the number of early or late type stars, but have been treated as an extra class of objects. Objects in this class can be of different type: highly extincted background objects (and thus not very relevant for an analysis of the central parsec), sources with dusty envelopes, e.g. IRS 3 (Pott et al. 2008), young stars, whose strong winds interact with the interstellar medium in the form of bow-shocks (Tanner et al. 2002, 2003), or even candidates for young stellar objects (Muzic et al. 2008) (examples for the SEDs of these sources are shown in Fig. 18). Table 5 lists 
Table 5. Stars classified in the GC using the method described in Sect. 2.7.

\begin{tabular}{cc}
\hline \hline Class & Number \\
\hline early type quality 1 & 277 \\
early type quality 2 & 25 \\
early type quality 3 & 10 \\
late type quality 1 & 2955 \\
late type quality 2 & 2231 \\
foreground & 58 \\
very red stars & 24 \\
noisy sources & 334 \\
\hline
\end{tabular}

the stars in each (sub)category, while Fig. 10 shows the spatial distribution of the classified sources.

\subsection{Comparison with spectroscopic results and uncertainty estimation}

We compared the results of our classification to the lists of spectroscopically classified sources provided by Paumard et al. (2006) and Maness et al. (2007). The list published by Paumard et al. (2006) contains 90 early type stars, 78 of which were contained in our list of common sources. This discrepancy can be explained by the nature of the observations used here (different observation dates for the individual filters, 2 years between $H$ band and IB observations, different quality of the datasets), some sources (especially fast moving objects like S2) were not detected at the same position in every image, not detected through all filters or excluded due to too large photometric uncertainties and thus did not make it on the common list. The same effects are probably relevant for the 329 sources classified by Maness et al. (2007), while in addition, parts of this sample are outside of the area covered by our data. We only used the 266 sources that are also present in our dataset for comparison.

67 of the 78 known early type sources were also classified as early type here, 7 had too noisy SEDs and 4 were classified as late type. Of these 4 sources, 3 are borderline cases where a clear identification is very difficult with our method (sources 224, 612 and 1772 resp. E36, E89 and E7 in the Paumard list). Source 3778 (E37 in the Paumard list) shows a clear CO absorption feature. Paumard et al. (2006) classify this source as a potential O8-9 supergiant, but at the same time give it a $K$ band magnitude of 14.8 and an absolute magnitude of -3.3 . This is inconsistent with our expectations, since such a source should be at least two magnitudes brighter (see Table 3). We therefore ignore this source for the uncertainty estimation. This leads us to 3 out of 77 sources classified erroneously and 7 out of 77 sources not classified, which corresponds to $3.9 \%$ respectively $9.1 \%$. A few well known sources like IRS $16 \mathrm{SW}$ and IRS 15NE have noisy SEDs, which in the case of IRS $16 \mathrm{SW}$ is probably due to the intrinsic variability of that source. But in general, noisy SEDs mostly stem from problems with the photometry: here, too faint or saturated sources are the biggest issues.

Since the known early type sources are concentrated in the inner $0.5 \mathrm{pc}$, these values can be adopted as the uncertainties of the number of early type stars in the innermost few arcseconds identified in this work.

258 of the 266 known late type sources have been classified as late types by our method. The SEDs of 7 sources were too noisy and one was classified as early type (source 363, 96 in Maness list). Source 363 does not show a clear CO feature despite being bright enough, and a comparison of the Maness and Paumard lists shows that there is an early type source $0.17^{\prime \prime}$ from its position (assuming that the positions given in these works use the same reference frame). In the imaging data that we have used for this work, there is only a single source present at the location of source E87 (Paumard) resp. 96 (Maness). It appears sufficiently isolated to rule out confusion with another source. This leads us to the assumption that Paumard et al. (2006) and Maness et al. (2007) are looking at the same source there, but classify it differently.

In order to derive an upper limit for the uncertainty and thus the confidence in our method, we assume one erroneous classification in the area covered by the SINFONI observations. In this region (north of $\mathrm{Sgr} \mathrm{A}^{*}$, mostly outside of $0.5 \mathrm{pc}$ ), we find a total number of 11 sources classified as early type, including the one star of disputed type. If we assume one of these classifications to be erroneous, this leads us to an uncertainty of $\sim 9 \%$ for our number of early type stars outside of $0.5 \mathrm{pc}$. We consider this an acceptable level of confidence, considering the low density of early type stars we measure this far out.

To be on the conservative side, we adopt this value, $\pm 9 \%$, as the uncertainty of the total number of all early type stars, i.e. in the entire field of view. It has to be considered, however, that the low total number of sources available for the determination of this uncertainty level limits the confidence in it.

\subsection{Structure of the cluster}

Figure 11 shows the projected stellar density for the total population, the early and late type stars. For comparison, we also show the projected density of the early type stars provided by Paumard et al. (2006). Only stars brighter than 15.5 mag have been considered here, in order to allow a clear separation of early and late type stars and to make a completeness correction unnecessary (see Schödel et al. 2007).

The projected density profile of the late type stars is practically flat within a radius of $\sim 10^{\prime \prime}$. Within the innermost $5^{\prime \prime}$, it can even be fitted with a power law with a positive slope, i.e. the projected density increases with the distance to the center. This flattening or even inversion of the projected surface density profile of the late-type stars combined with the steeply increasing density of early-type stars towards Sgr A* explains the dip in CO band head absorption strength found in early spectroscopic observations of low spatial resolution (Allen et al. 1990; Sellgren et al. 1990; Haller et al. 1996). We discuss this in detail in Sect. 3.4.

A dip in the density can be observed at a radius of $\sim 5^{\prime \prime}$ that has already been observed by Schödel et al. (2007) in deep $\left(\operatorname{mag}_{K} \leq 17.5\right)$ star counts. Zhu et al. (2008) also find a dip at $0.2 \mathrm{pc}$, which corresponds to the $5^{\prime \prime}$ given here and in Schödel et al. (2007). This dip is a significant feature in the density profiles. It is both present in the late- and early-type population, although with a low significance in the latter, due to the small number of early-type stars. The cause for this feature is probably extinction, since there is a ring-like area of high extinction visible in our extinction map at this distance to the center (see Fig. 17). Since this dip feature seems to appear in two stellar populations that are so different in their age and their dynamical state and since it would be very difficult to reproduce such a feature in a three-dimensional distribution, extinction seems to be the most likely explanation.

A steep increase of the projected density of early type stars can be observed towards the center (except in the innermost arcsecond). This also confirms previous observations. Paumard et al. (2006) proposed that the projected density of the early type stars follows a $R^{-2}$ power law outside of a sharp inner edge at $1^{\prime \prime}$ 
(within the $\operatorname{disk}(\mathrm{s})$, so this value is not directly comparable to our findings), with $R$ as the projected distance to $\operatorname{Sgr} \mathrm{A}^{*}$. Lu et al. (2009) also confirmed the $R^{-2}$ power law within the clockwise disk. To allow a comparison with our values, we fitted the projected density of the known early type sources that are contained in our sample with a power las as well. This yielded a value of $\beta_{\text {ref }}=-1.80 \pm 0.17$. In the following, we adopt this value as a reference.

We fitted the projected early type density with a power law $\rho_{\text {proj }} \propto R^{\beta_{1 \prime}}$, excluding the inner $1^{\prime \prime}$. But while providing a value that agrees well with previously published results (Genzel et al. 2003; Paumard et al. 2006; Lu et al. 2009), this single power law does not provide a very good fit to our data. For comparison, we also fitted the projected early type density with a broken power law with a break at $10^{\prime \prime}$. This minimizes the deviations from the data, but it introduces a break at $10^{\prime \prime}$ that we cannot explain.

$$
\begin{aligned}
\beta_{1^{\prime \prime}} & =-1.49 \pm 0.12 \\
\beta_{1-10^{\prime \prime}} & =-1.08 \pm 0.12 \\
\beta_{10-20^{\prime \prime}} & =-3.46 \pm 0.58 .
\end{aligned}
$$

It has to be kept in mind that the absolute number of early type stars in the outer regions is much lower than further towards the center, so the uncertainties are considerably larger. The value for $R>1^{\prime \prime}$ agrees reasonably well with the values determined by Genzel et al. (2003); Paumard et al. (2006); Lu et al. (2009). At $R<1^{\prime \prime}$, the early type density is lower than expected from extending the power law inwards. This is the sign of the inner edge of the clockwise disk reported by Paumard et al. (2006) and Lu et al. (2008). In this densest part of the cluster, the stellar surface density is probably also underestimated because source confusion will lead to incompleteness of the data (we estimate that completeness of sources at $\operatorname{mag}_{K} \leq 15.5$ drops to $\sim 80 \%$ within $0.5^{\prime \prime}$ of $\left.\mathrm{Sgr} \mathrm{A}^{*}\right)$. Outside of $1^{\prime \prime}$, we detect a number of early type stars (312 over the whole field compared to 90) that is by a factor of $\sim 3$ higher than in previous works. Their density profile is similar to that of the previously reported early-type sources. The power law in the inner few arcseconds becomes flatter with the additional early-type stars included, but agrees within the uncertainties with the previously reported values. Towards the edges of the cluster $\left(\sim 10-20^{\prime \prime}\right)$, we observe a steeper density profile than Paumard et al. (2006). It may be possible that a different density law applies outside of $10^{\prime \prime}$, but the cause for such a phenomenon is unknown. It has to be considered, however, that the statistics for such a small number of stars at larger distances are not very reliable any more. In addition, two different cutoffs were used for sources inside and outside of $12^{\prime \prime}$. This can also lead to a bias here in the way that the early type density is underestimated outside of $12^{\prime \prime}$ and overestimated within that distance to $\mathrm{Sgr} \mathrm{A}^{*}$. But this effect should not influence the density in the order of magnitude observed here which leads to the different slopes of the fitted power laws.

The relative stellar density of the sources rated as too noisy (see Fig. 9 appears to be flat over an inner region with a radius of $\sim 15^{\prime \prime}$. Outside of $15^{\prime \prime}$, the ratio increases, but that can be expected since the quality of the photometry decreases somewhat toward the edge of the FOV. This is probably due to the rectangular dither pattern used that leads to shallower integration toward the edges of the FOV.

This gives further evidence that our criteria for excluding noisy sources as well as the local calibration we apply are justified, since an even distribution of noisy sources can be expected from a well calibrated dataset. This is the case here, and it means percentage of noisy sources

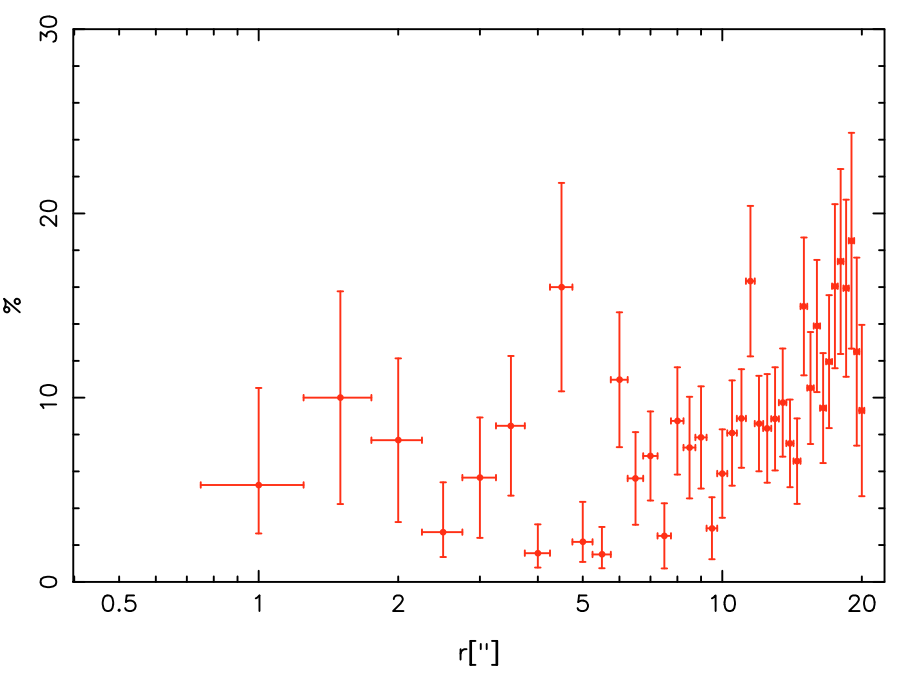

Fig. 9. Azimuthally averaged density of stars with noisy SEDs. The density profile is practically flat over the inner $15^{\prime \prime}$, with a slight increase further out.

that the exclusion of noisy sources does not lead to a bias in our surface density profiles.

Figure 12 shows the two-dimensional density distribution of all stars brighter than $\operatorname{mag}_{K}=15.5$ and Fig. 13 that of the late type stars of the same magnitude range, while Fig. 14 shows the density of the early type stars in the same way. These maps have been smoothed with a $\sim 4$ " Gaussian. While the late type stars show a similar distribution as the stars in the cluster viewed as a whole (with the exception of the central few arcseconds), the early type stars are concentrated in the center. This result is not surprising since the same can be seen from the azimuthally averaged density. The area in the immediate vicinity of IRS 7 shows a significantly lower stellar density in all our maps, because the presence of this extremely bright source impedes the detection of other stars close to it.

The peak of the early type density is, as expected, located close to the position of $\operatorname{Sgr} A^{*}$. The distribution of the early type stars appears close to circularly symmetric, but indicates extensions along N-S and E-W. An apparent concentration of early-type stars along these directions can also be seen in Fig. 1 of Bartko et al. (2009).

The density distribution of the late type stars clearly shows the relative lack of late type stars in the very center. The map also shows a correlation with the extinction map (see extinction map provided in Schödel et al. 2007; and Fig. 17). Areas of higher extinction show a lower density of stars. This effect is not visible in the early type density. But in the areas with higher extinction (e.g. $5^{\prime \prime} \mathrm{NW}$ of Sgr A*), the early type density is too low for this effect to be relevant, although a trace of it can be seen in the slight dip in the early type density in Fig. 11. The extinction has two separate effects on the projected density: one effect is that sources behind a lot of extinction appear fainter, so they might be excluded by a simple magnitude cutoff. This effect has been corrected here by using extinction corrected magnitudes. The other problem is that extinction also impedes the detection of sources, especially in filters with lower image quality. This effect of the extinction on the stellar density has not been calculated here and was not compensated. 


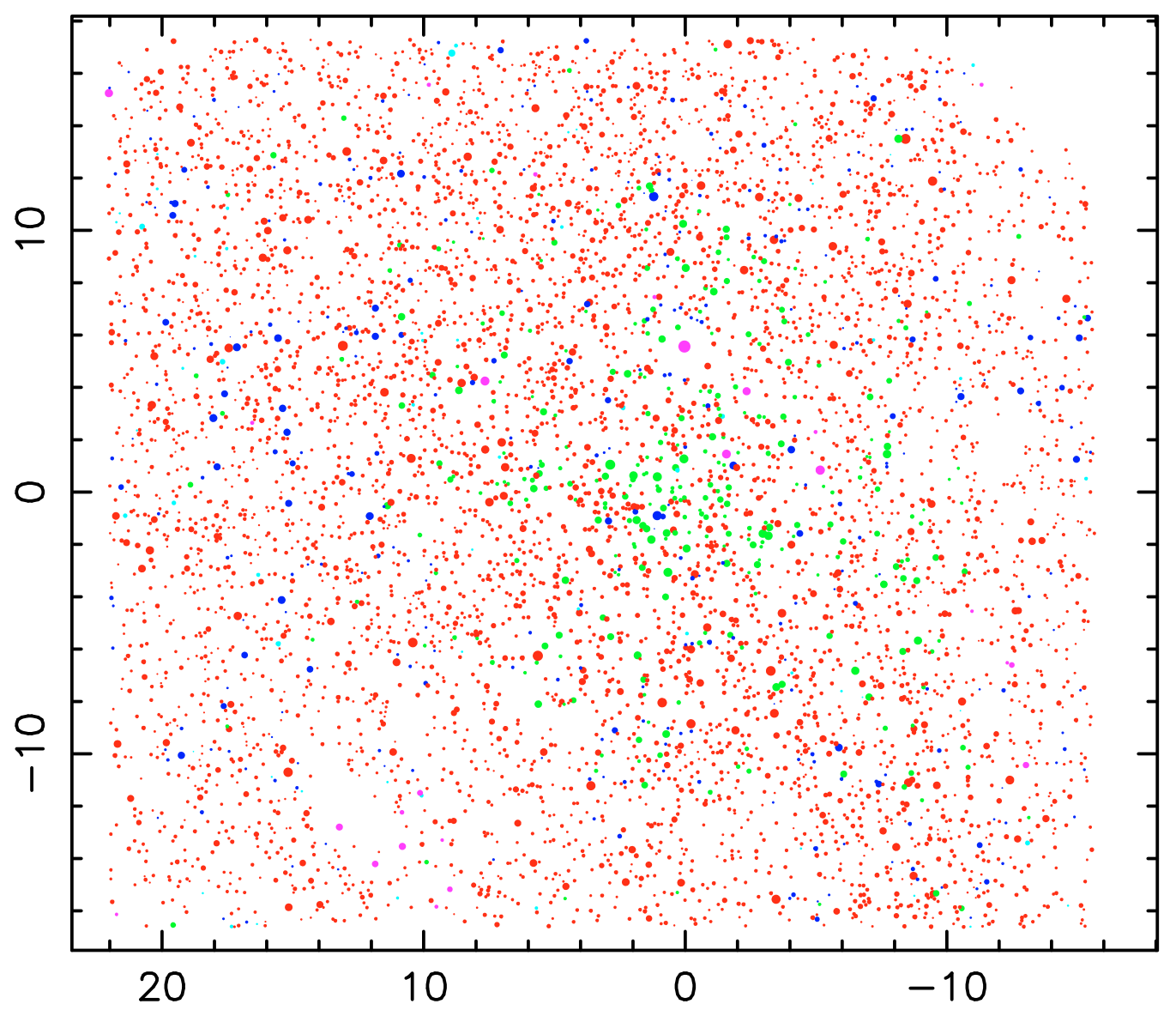

Fig. 10. Map of the stars in the GC. Red: late type stars. Green: early type candidates. Blue: sources not classified. Light blue: foreground sources. Magenta: extremely red objects. The radii of the circles are linearly dependent on the extinction corrected magnitudes of the sources.

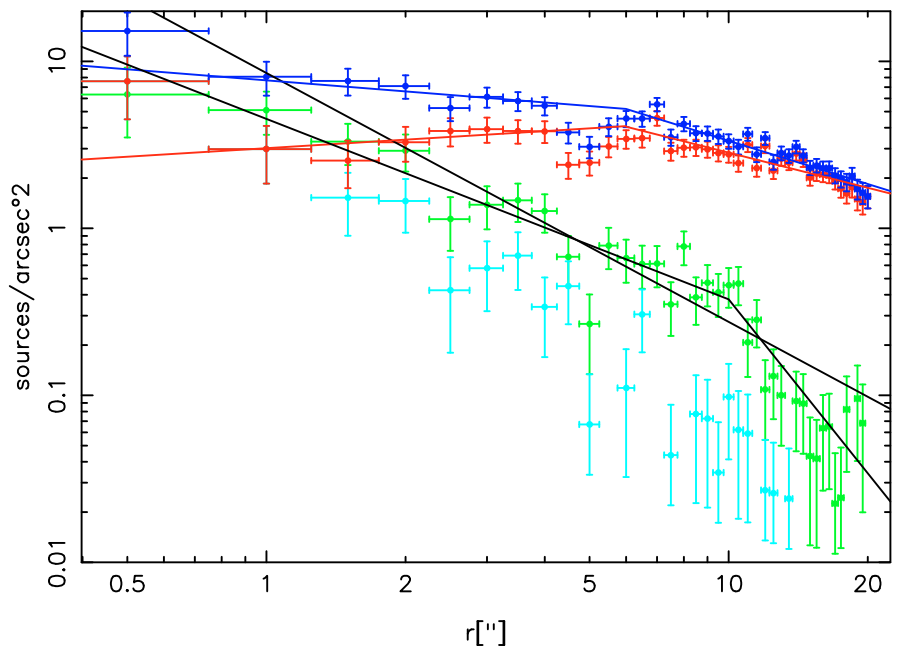

Fig. 11. Azimuthally averaged stellar surface density plotted against the distance to $\mathrm{Sgr} \mathrm{A} *$ for $K \mathrm{~s}$ magnitudes $\operatorname{mag}_{K \mathrm{~s}}<15.5$. The green points describe the distribution of early type stars, while red stands for late type quality 1 stars and dark blue for all detected stars. This also includes stars rated as noisy and bright enough late type quality 2 sources. The early type stars given in Paumard et al. (2006) are shown in light blue for comparison. The solid lines indicate the power laws fitted to the data.

\subsection{Evidence for giant depletion in the center}

Figer et al. (2003) examined the radial velocities of 85 cool stars in the GC (mostly M and $\mathrm{K}$ giants) and found dynamical

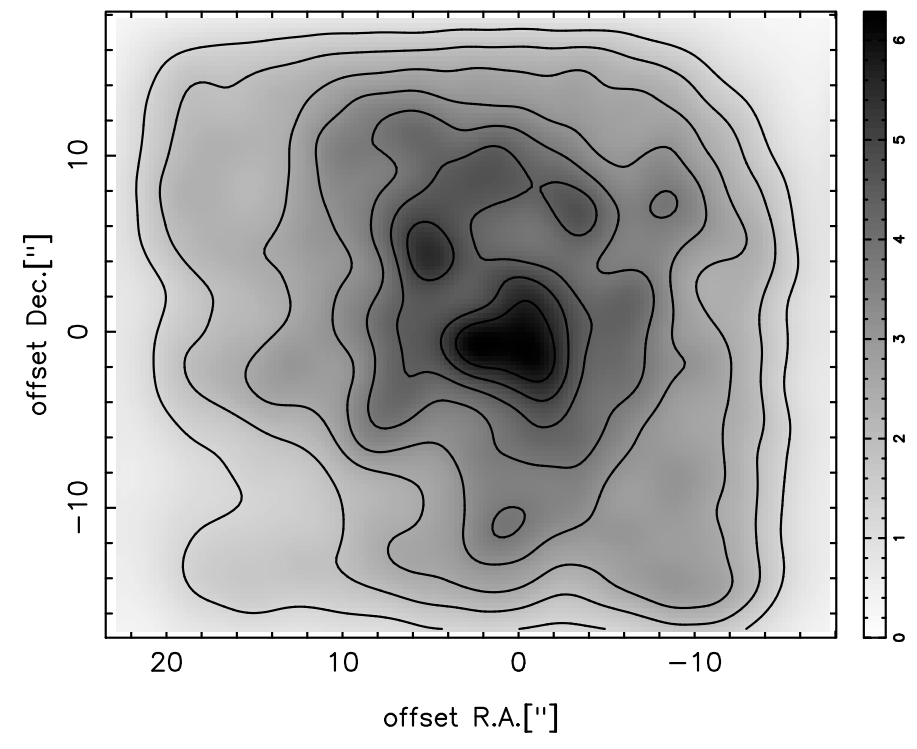

Fig. 12. Stellar surface density of all stars brighter than 15.5 mag (linear scale, contours trace density in steps of $20,30 \ldots 90$ percent of maximum density). The density is given in units of sources per $\operatorname{arcsec}^{2}$.

evidence for a flattened distribution of late type stars within 0.4 pc $\left(\sim 10^{\prime \prime}\right)$. As Figer et al. (2003) and Zhu et al. (2008) point out, the flat projected surface density profile of the late-type stars implies in fact a hole/dip in the 3-dimensional distribution of the late-type stars. 


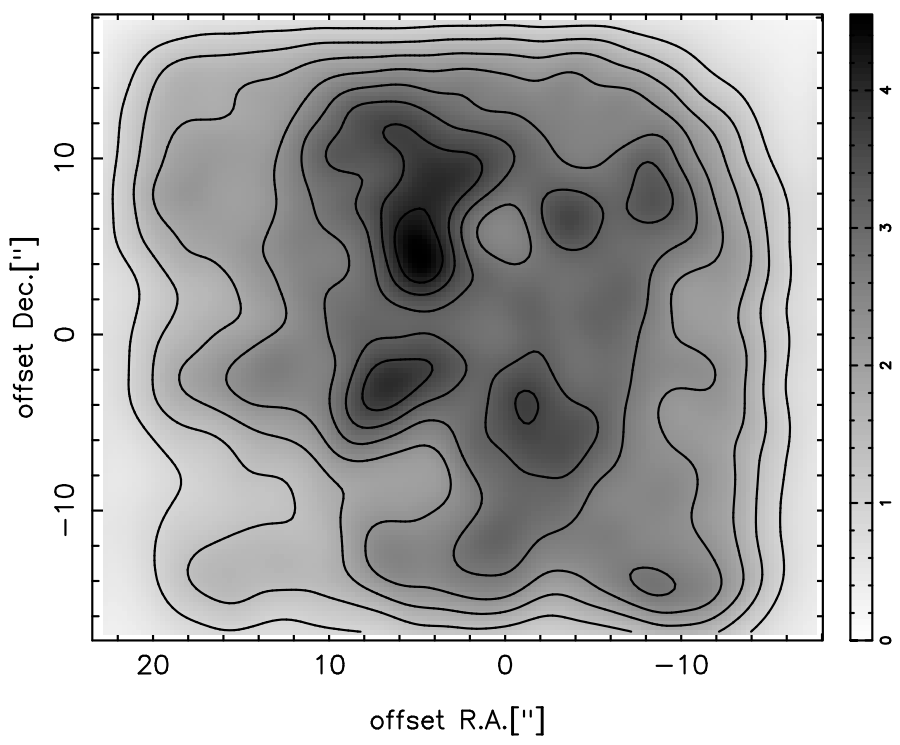

Fig. 13. Stellar surface density of late type stars brighter than $15.5 \mathrm{mag}$ (linear scale, contours trace density in steps of $20,30 \ldots 90$ percent of maximum density). The density is given in units of sources per $\operatorname{arcsec}^{2}$.

Schödel et al. (2007) described the total population of the central parsec with a broken power law (break radius $R_{\text {break }}=$ $6^{\prime \prime} .0 \pm 1^{\prime \prime} .0, \beta=-0.19 \pm 0.05$ within $R_{\text {break }}$ and $\beta=-0.75 \pm$ 0.10 outside of the break radius) for a magnitude limit of 17.75 and using completeness corrected data. This does not allow a comparison of absolute densities to our findings, since that dataset goes much deeper than ours, but the trend can be confirmed here. It also has to be considered that these results were obtained on the entire population and not separated into early and late type stars.

We fitted broken power laws to the projected densities of the late type stars and all classified stars (see Table 6). We find the same break radius of $6.0 \pm 1.0^{\prime \prime}$ as Schödel et al. (2007), and the power law indices for the total population also agree with the values given in that work $(-0.22 \pm 0.11$ for the inner region, $-0.86 \pm 0.06$ for $R>6^{\prime \prime}$ in our data). What is new here, however, is the possibility to obtain separate values for the late type population alone. These values give an even stronger support to the proposed hole/dip in the center: we find a power law index of $-0.70 \pm 0.09$ for the outer region, while the inner region even shows a decline towards the center $(0.17 \pm 0.09)$.

This is a very interesting result, since it shows that the previously observed flattening of the density profile of the total population (Genzel et al. 1996; Figer et al. 2003; Genzel et al. 2003; Schödel et al. 2007; Zhu et al. 2008) is an even stronger feature in the late type population. We can therefore assume that the stellar population in the innermost $\sim 0.2 \mathrm{pc}$ is indeed depleted not only of bright giants, but also of fainter giants down to our magnitude limit of 15.5 mag. Several causes for this have been proposed: Dale et al. (2008) offered an explanation for the underdensity of late type stars within $1^{\prime \prime}$, claiming that collisions with stellar mass black holes and main sequence stars prevent $1-2 M_{\odot}$ giants to evolve so that they are not visible in the $K$ band. Their simulations cannot explain the lack of brighter and fainter giants. Freitag et al. (2008) derived collision probabilities for bright giants in the GC (see esp. their Fig. 1). They determined that nearly all massive stars within $0.1 \mathrm{pc}$ almost certainly suffer from collisions during their time on the giant branch.

Merritt \& Szell (2006) offered yet another explanation: the infall of a second SMBH would destroy the stellar density cusp

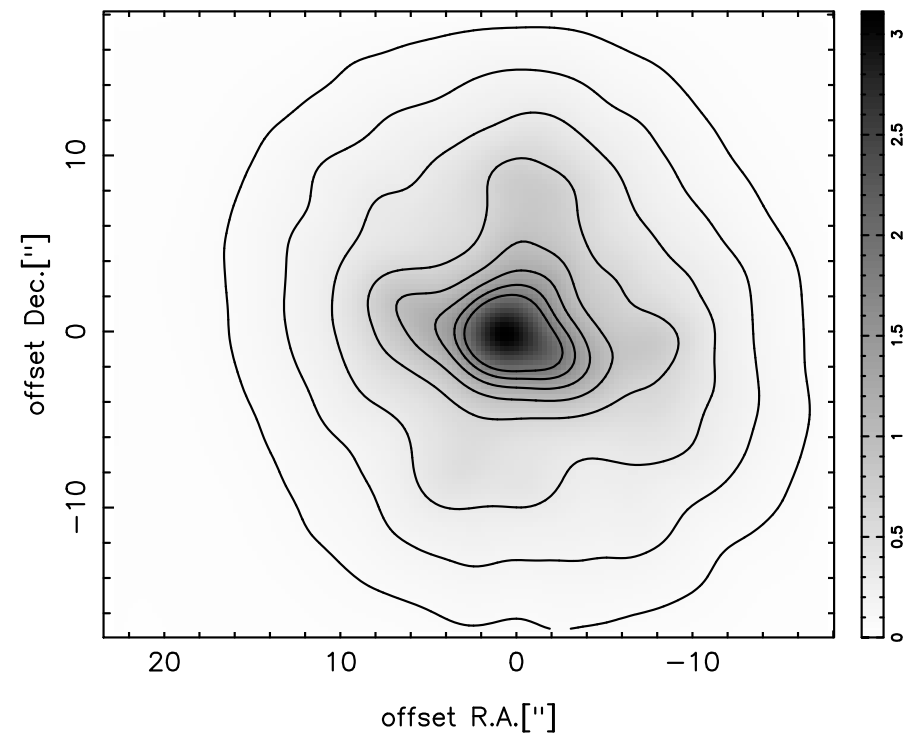

Fig. 14. Stellar surface density of early type stars brighter than 15.5 mag (logarithmic scale, contours trace density in steps of 2.5, 5, 10, 20 ... 90 percent of maximum density). The density is given in units of sources per $\operatorname{arcsec}^{2}$.

present around Sgr A*, which would then be built up again in a time-frame of several Gyrs. This process can lead to a practically flat density profile, similar to the one observed here.

\section{5. $K$ band luminosity function}

The general $K$ band luminosity function (Fig. 15) agrees well with the one presented by e.g. Genzel et al. (2003); Schödel et al. (2007). The red clump can clearly be made out at the expected magnitude of $\sim 15.0-15.25$. The 15.5 mag limit of the observations does not have a significant effect on this feature, since this limit only applies to the separation of early and late type stars, while the photometric completeness limit lies at $\sim 16$ mag.

The luminosity functions can be described to the first order by a power law (fitted to the area between 9.0 and 15.5 mag for the early type stars, resp. 14.5 to exclude the red clump in the other plots):

$\frac{\mathrm{d} \log N}{\mathrm{~d} K}=\beta$

The power law indices that resulted from this fit are shown in Table 7. Alexander \& Sternberg (1999); Tiede et al. (1995); Zoccali et al. (2003) measured the power law slope of the bulge population of the milky way several degrees from the center as $\beta \sim 0.3$, while Figer et al. (2004) give the same value for the KLF on $30 \mathrm{pc}$ scales around the GC, claiming that this value agrees very well with the theoretical KLF of an old stellar population, reflecting the rate of evolution of stars along the red giant and asymptotic giant branch. Genzel et al. (2003) give a value of $\beta=0.21 \pm 0.02$ for the central $9^{\prime \prime}$. It is expected that for greater distances to the center, the slope value approaches that of the bulge.

The power law fitted here for the total population of the central parsec is flatter than the one attributed to the bulge population, while our value fitted for the inner $9^{\prime \prime}$ matches the value of 0.21 given by Genzel et al. (2003).

The individual KLFs for the late and early types give the reason for this deviation from the bulge power law: since the central parsec (and even more the central few arcseconds) contain a 

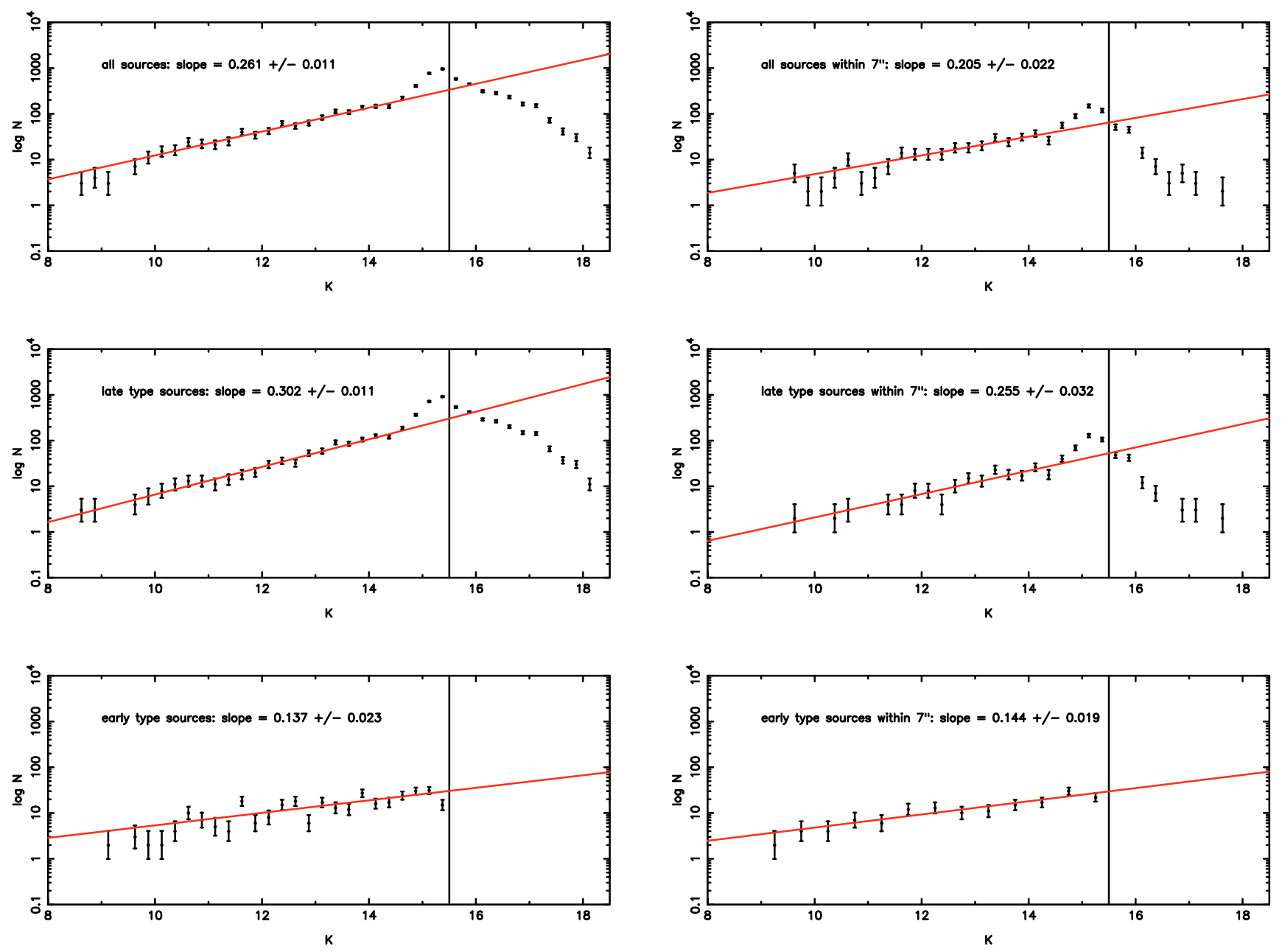

Fig. 15. Luminosity function of stars in the central parsec. Each data-point represents the center of a magnitude bin ( 0.25 mag wide bins, resp. 0.5 mag in lower right frame). Upper left: all stars. Central left: only late type stars. Lower left: only early type stars. Upper right: all stars within 7". Central right: late type stars within 7". Lower right: early type stars within 7".

Table 6. Power law indices for late type stars and all classified stars, separate fit to sources inside and outside of $6.0^{\prime \prime}$.

\begin{tabular}{lrr}
\hline \hline & $\beta_{\text {inner }}$ & $\beta_{\text {outer }}$ \\
\hline all stars & $-0.22 \pm 0.11$ & $-0.86 \pm 0.08$ \\
late type stars & $0.17 \pm 0.09$ & $-0.70 \pm 0.09$ \\
\hline
\end{tabular}

Table 7. KLF power law indices of the different classified stellar types, calculated for the entire cluster resp. the inner $7^{\prime \prime}$.

\begin{tabular}{lcc}
\hline \hline Type & Complete cluster & $r<7^{\prime \prime}$ \\
\hline all & $0.26 \pm 0.01$ & $0.21 \pm 0.02$ \\
late & $0.31 \pm 0.01$ & $0.27 \pm 0.03$ \\
early & $0.14 \pm 0.02$ & $0.13 \pm 0.02$ \\
\hline
\end{tabular}

significant number of early type stars and their KLF has a much flatter slope $\left(\beta_{\text {early }}=0.14 \pm 0.02\right)$, the resulting power law is also flatter than that of the late type population alone. The power law fitted to the KLF of only the late type stars $\left(\beta_{\text {late }}=0.31 \pm 0.01\right)$ agrees very well with the one observed in the bulge.

Agreeing with Lebofsky \& Rieke (1987); Blum et al. (1996); Davidge et al. (1997); Genzel et al. (2003), our data point to the population in the central parsec being an old stellar population with an admixture of a young, bright component.
The fitted slope value for the inner $9^{\prime \prime}$ of 0.21 differs from the one for the total cluster, but otherwise, the shape of the KLF there is very similar. This difference is due to the flatter slope of the late type KLF in this region, while that of the early type KLF stays the same.

Paumard et al. (2006) also presented a KLF for the early type stars in the disks. They also find a flat KLF, similar to the one presented here. The significant difference is the greater sensitivity we achieved here. Paumard et al. (2006) note that their KLF has a spectroscopic completeness limit of $13.5-14$ mag, compared to $15.5 \mathrm{mag}$ here. The fact that the flat slope of the early type KLF can be observed down to that magnitude is an important new result and it strengthens assumptions of a top-heavy mass function since it improves the statistic relevance of the observed flatness.

\subsection{Extinction}

The average extinction of 3.3 mag towards the GC is well known (e.g. Scoville et al. 2003), but this value varies on small scales by up to 1.5-2 mag (Schödel et al. 2007) and poses a significant problem for the reliable measurement of apparent magnitudes, colors and intrinsic reddening of stars. Schödel et al. (in preparation) are presenting an extinction map based on $H-K$ colors obtained from the same data that we are using here, but 
source 355
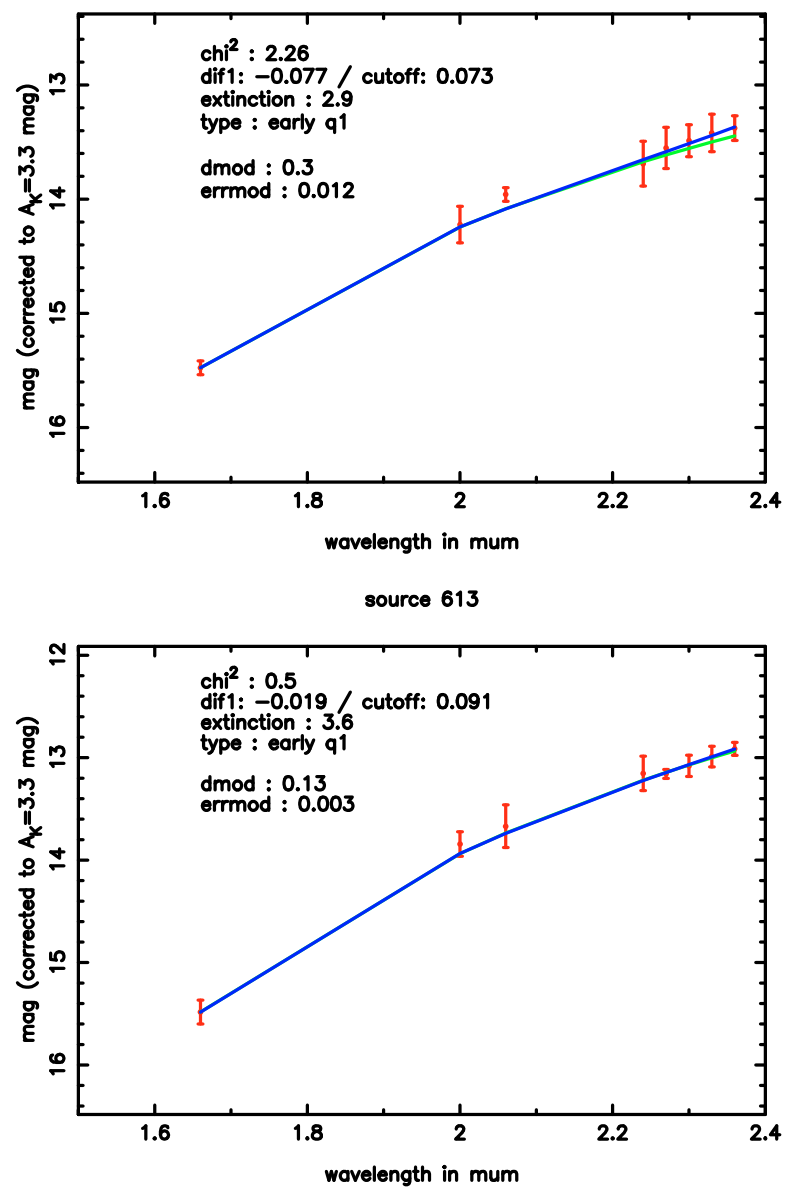

Fig. 16. SEDs of stars fitted as early type outside of $0.5 \mathrm{pc}$.

the individual extinction values obtained here can also be used to produce an extinction map. Figure 17 shows this map. It agrees well with Schödel et al. (2007), except that we find higher overall extinction values. The extinction map produced in this work is expected to be more reliable since we use many wavelength bands, apply a local calibration and distinguish between hot and cool sources. A histogram of the measured extinction values is shown in Fig. 17: the distribution is similar to a Gaussian with a mean value and standard deviation of $3.1 \pm 0.4$. This agrees well with previously published results (Scoville et al. 2003) and also with Schödel et al. (in preparation). The asymmetry of the histogram is due to the exclusion of foreground stars.

It has to be noted, however, that the Draine (1989) extinction law was used here and that a different law might apply to the GC. This may lead to a systematic offset as large as $A_{K}=0.5$ mag, which should be taken into account as a possible systematic uncertainty when interpreting these values.

\subsection{Early type stars outside of $0.5 p c$}

In addition to not only identifying the well known early type stars in the center of the cluster, but indeed more than tripling the number of early type candidates in the central $0.5 \mathrm{pc}$, we can also report the identification of 35 early type candidates more than $0.5 \mathrm{pc}\left(\sim 12.9^{\prime \prime}\right)$ from Sgr A*. If we apply our previously derived uncertainty of $\sim 9 \%$ (see Sect. 3.2), this leads us to a number of $35 \pm 3$ early type candidates outside of the inner region. This number is most likely underestimated, due to the stricter source 363
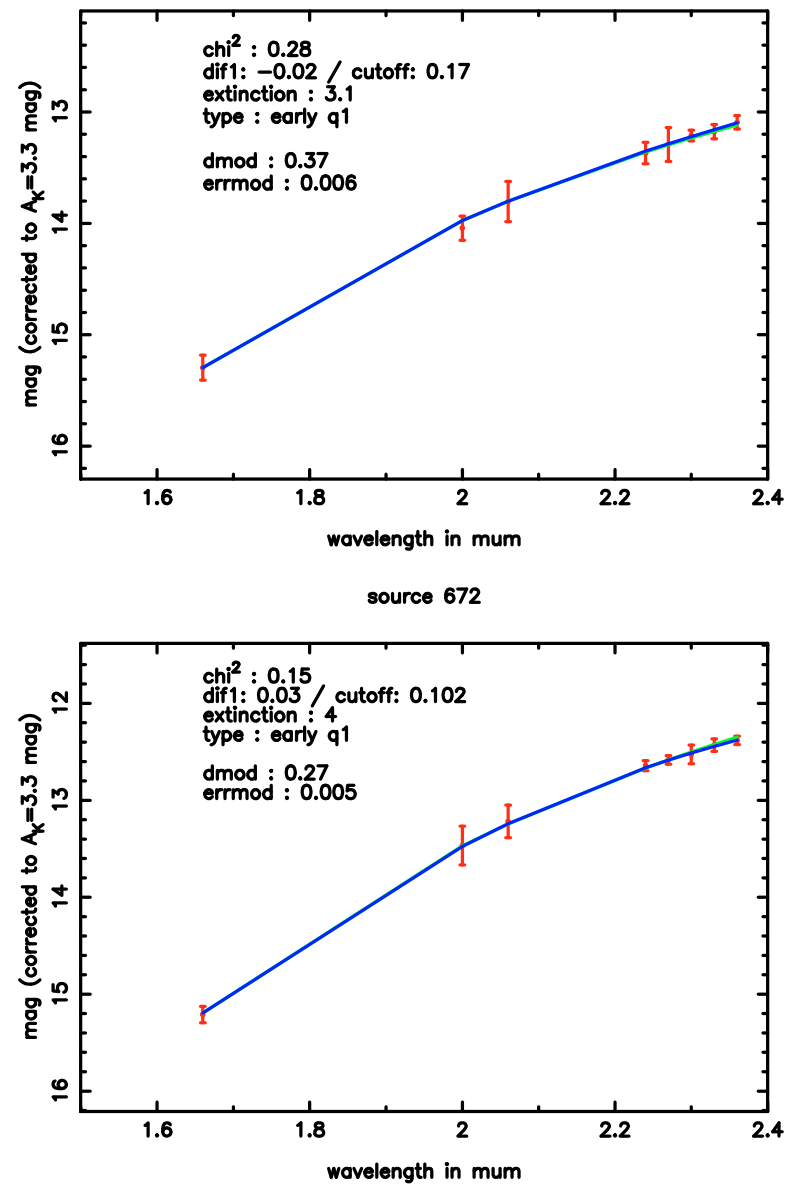

cutoff criterion used in this region (see Sect. 2.5). Within the observed area, the distribution of these sources appears to be fairly isotropic, although one has to be cautious here due to the the asymmetry of the observed region and the small number of candidates. The nature of these candidates needs to be confirmed with spectroscopic observations, since our method can only provide a first estimate for the type.

Figure 16 shows four examples of what we consider very likely candidates for actual early type stars. The source in the upper right frame of Fig. 16 does not show a $\mathrm{CO}$ absorption feature, but still it has been labeled as a late type source in Maness et al. (2007). Paumard et al. (2006) seem to list it as an early type however. The other sources listed in Table 8 with quality A have similarly smooth SEDs, while quality B and C sources are considerably more noisy.

We obtain an average density of early type stars of (4.6 \pm $0.4) \times 10^{-2}$ sources/ $\operatorname{arcsec}^{2}$ at $R>12.9^{\prime \prime}$.

The detection of early type stars this far out in significant numbers is a new result: Paumard et al. (2006) reported no early type stars outside of the central $0.5 \mathrm{pc}$, citing an $1 \sigma$ upper limit of $\sim 10^{-2}$ OB stars per $\operatorname{arcsec}^{2}$ outside of $13^{\prime \prime}$ deduced from SINFONI data, but referring to an unpublished source. The difficulty in identifying these stars in existing data is pointed out by Trippe et al. (2008), who mention very limited coverage of the central cluster and data gathered with several instruments and in different epochs that is very difficult to compare due to different pixel scales, Strehl ratios and completeness. These obstacles have probably impeded the detection of early type stars in the 

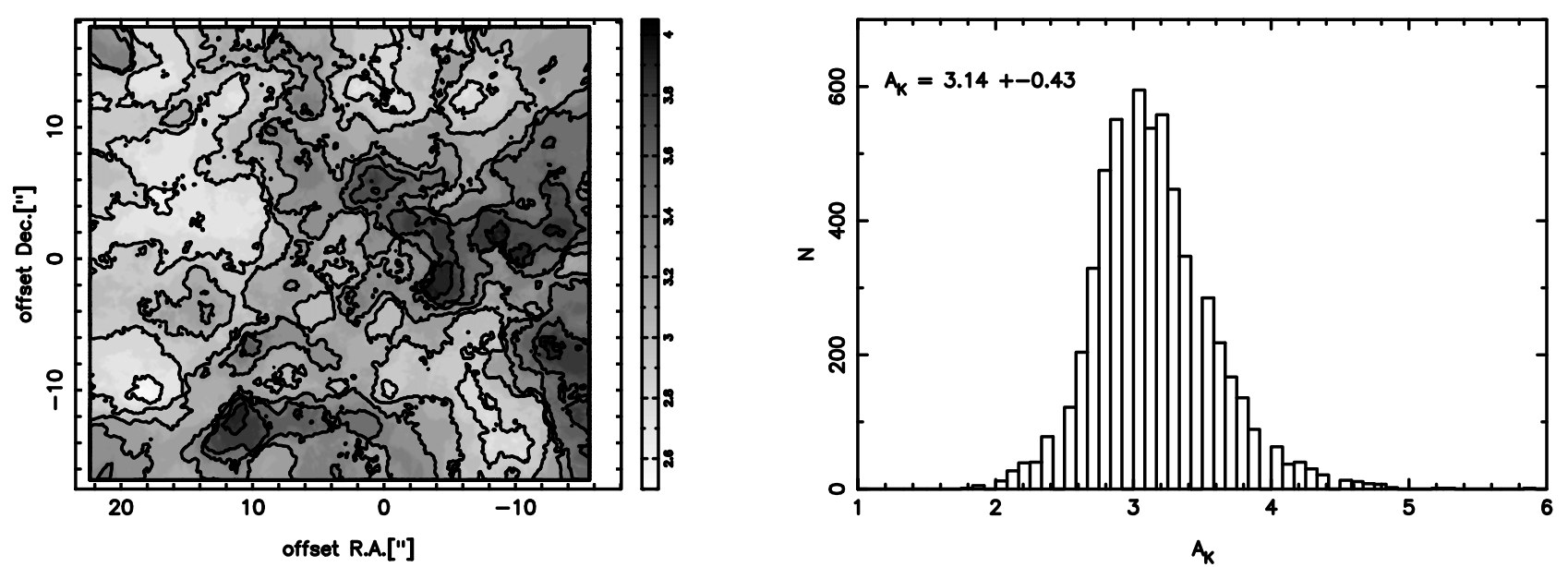

Fig. 17. Left: extinction map of the central parsec computed from individually fitted extinction values. Features like the mini-cavity and the minispiral are visible. Right: distribution of extinction values in the central parsec of the GC.

Table 8. Early type stars detected outside of 0.5 pc. $x$ and $y$ denote the position of the star in $\operatorname{arcsec}$ relative to $\mathrm{Sgr} \mathrm{A}^{*}, \operatorname{mag}_{K \text { ext }}$ the extinction corrected $K$ band magnitude and $A_{K}$ the extinction in the $K$ band. Quality indicates the confidence in the identification after an additional visual inspection, with $A$ as the highest confidence and $\mathrm{C}$ as the lowest.

\begin{tabular}{cccccc}
\hline \hline$x\left({ }^{\prime \prime}\right)$ & $y\left(^{\prime \prime}\right)$ & $\mathrm{mag}_{K, \text { ext }}$ & $A_{K}$ & CBD & Quality \\
\hline-8.15 & 13.50 & 10.7 & 3.3 & $0.07 \pm 0.04$ & $\mathrm{C}$ \\
-9.59 & -15.33 & 12.5 & 3.1 & $-0.05 \pm 0.01$ & $\mathrm{~B}$ \\
15.74 & 12.87 & 13.1 & 2.8 & $0.03 \pm 0.03$ & $\mathrm{~B}$ \\
-8.37 & -11.27 & 13.9 & 2.7 & $-0.095 \pm 0.019$ & $\mathrm{C}$ \\
-8.64 & -10.74 & 13.5 & 3.0 & $-0.003 \pm 0.003$ & $\mathrm{~A}$ \\
-10.57 & -15.90 & 13.0 & 3.3 & $0.05 \pm 0.01$ & $\mathrm{~B}$ \\
18.92 & 0.28 & 13.3 & 3.2 & $0.02 \pm 0.01$ & $\mathrm{~B}$ \\
-10.62 & -9.76 & 13.2 & 3.3 & $0.008 \pm 0.017$ & $\mathrm{~A}$ \\
-10.81 & -10.51 & 14.1 & 3.0 & $0.01 \pm 0.01$ & $\mathrm{~B}$ \\
13.05 & 14.29 & 13.3 & 3.5 & $0.04 \pm 0.02$ & $\mathrm{~B}$ \\
7.39 & 12.29 & 13.3 & 3.5 & $0.03 \pm 0.01$ & $\mathrm{~A}$ \\
13.13 & 5.08 & 14.6 & 2.8 & $-0.07 \pm 0.04$ & $\mathrm{~B}$ \\
19.58 & -16.54 & 12.7 & 4.0 & $0.032 \pm 0.005$ & $\mathrm{~A}$ \\
4.43 & 16.11 & 13.9 & 3.4 & $0.028 \pm 0.007$ & $\mathrm{~B}$ \\
9.43 & 9.29 & 13.6 & 3.5 & $-0.008 \pm 0.018$ & $\mathrm{~B}$ \\
18.70 & 4.44 & 14.0 & 3.3 & $-0.08 \pm 0.03$ & $\mathrm{~B}$ \\
-10.86 & 7.20 & 13.3 & 3.8 & $0.05 \pm 0.02$ & $\mathrm{~B}$ \\
11.30 & 8.47 & 14.0 & 3.4 & $0.018 \pm 0.004$ & $\mathrm{~A}$ \\
17.69 & 3.01 & 14.0 & 3.4 & $0.03 \pm 0.01$ & $\mathrm{C}$ \\
10.91 & 9.44 & 11.9 & 4.7 & $-0.07 \pm 0.03$ & $\mathrm{~B}$ \\
-9.05 & -11.77 & 15.2 & 2.9 & $-0.089 \pm 0.005$ & $\mathrm{~A}$ \\
-12.75 & 9.77 & 12.8 & 4.4 & $0.05 \pm 0.02$ & $\mathrm{~B}$ \\
17.48 & 11.36 & 13.7 & 3.9 & $0.036 \pm 0.008$ & $\mathrm{~A}$ \\
-4.20 & 14.06 & 14.9 & 3.2 & $-0.08 \pm 0.02$ & $\mathrm{~B}$ \\
17.51 & -8.95 & 14.8 & 3.4 & $-0.07 \pm 0.01$ & $\mathrm{~A}$ \\
9.89 & -14.14 & 13.2 & 4.4 & $0.04 \pm 0.03$ & $\mathrm{~B}$ \\
14.25 & -5.64 & 14.5 & 3.8 & $-0.033 \pm 0.005$ & $\mathrm{~A}$ \\
12.55 & -4.19 & 13.2 & 4.7 & $0.03 \pm 0.02$ & $\mathrm{~A}$ \\
-15.25 & 6.68 & 14.1 & 4.1 & $-0.09 \pm 0.06$ & $\mathrm{~B}$ \\
-13.13 & -12.44 & 15.1 & 3.6 & $-0.079 \pm 0.004$ & $\mathrm{~A}$ \\
-5.14 & -15.75 & 15.4 & 3.4 & $-0.13 \pm 0.02$ & $\mathrm{C}$ \\
4.49 & -15.53 & 15.2 & 3.6 & $-0.14 \pm 0.03$ & $\mathrm{~B}$ \\
-1.16 & 16.91 & 13.8 & 4.5 & $-0.06 \pm 0.02$ & $\mathrm{~B}$ \\
0.31 & -15.30 & 15.1 & 3.7 & $-0.11 \pm 0.03$ & $\mathrm{~B}$ \\
8.86 & 9.55 & 15.4 & 3.5 & $-0.12 \pm 0.02$ & $\mathrm{C}$ \\
\hline & & & & &
\end{tabular}

outer region of the central cluster until now. Our new method can provide targets for spectroscopic confirmation observations, allowing for a broader search for early type candidates over a large area without the need to cover the whole area with integrated field spectroscopy. Finally, we would like to point out that we do know that at least one early type star exists outside of the central $0.5 \mathrm{pc}$, as has been confirmed by the results of Geballe et al. (2006) on IRS 8.

As Fig. 11 shows, there are two possible fitting solutions for the early type density. While the single power law fitted to the projected density of early type stars agrees with the previously published power law, the slopes of the broken power law show a significant difference in the outer and inner region. If a single power law distribution can be assumed, this would indicate that these stars merely represent the continuation of the disk and the off-disk population. But if the much steeper decline outside of $10^{\prime \prime}$ is indeed a significant and real feature, this might point to a change in the population respectively so far unknown effects on the density distribution.

\section{Conclusions}

Our newly developed method has confirmed several previously obtained results and has proven to be able to classify sources of known type reliably. It cannot compete with spectroscopic identifications of individual sources, but due to the ability to classify a large number of sources with a relatively small need for observation time, it can provide important statistical information about a stellar population which can later be refined by observing the early type candidates with spectroscopic methods. Our analysis extends the sensitivity limit of stellar classification by about 1.5 mag compared to previous work, providing thus a statistically stronger basis for conclusions on the stellar population.

The following results could be obtained:

1. A larger number of early type candidates has been detected than in any previous study, 312 sources compared to the known 90 . We were able to identify known early type sources with $87 \%$ accuracy, while $96 \%$ of the known late type sources were classified correctly. The different percentages stem from the different selection criteria of the reference sources in the cited publications: Maness et al. (2007) already selected their late type sources by the presence of sufficiently deep CO band heads, which is the same feature that our method makes use of. The early type sources published by Paumard et al. (2006) were selected based on narrow emission lines that are not visible at our spectral resolution. 
source 136
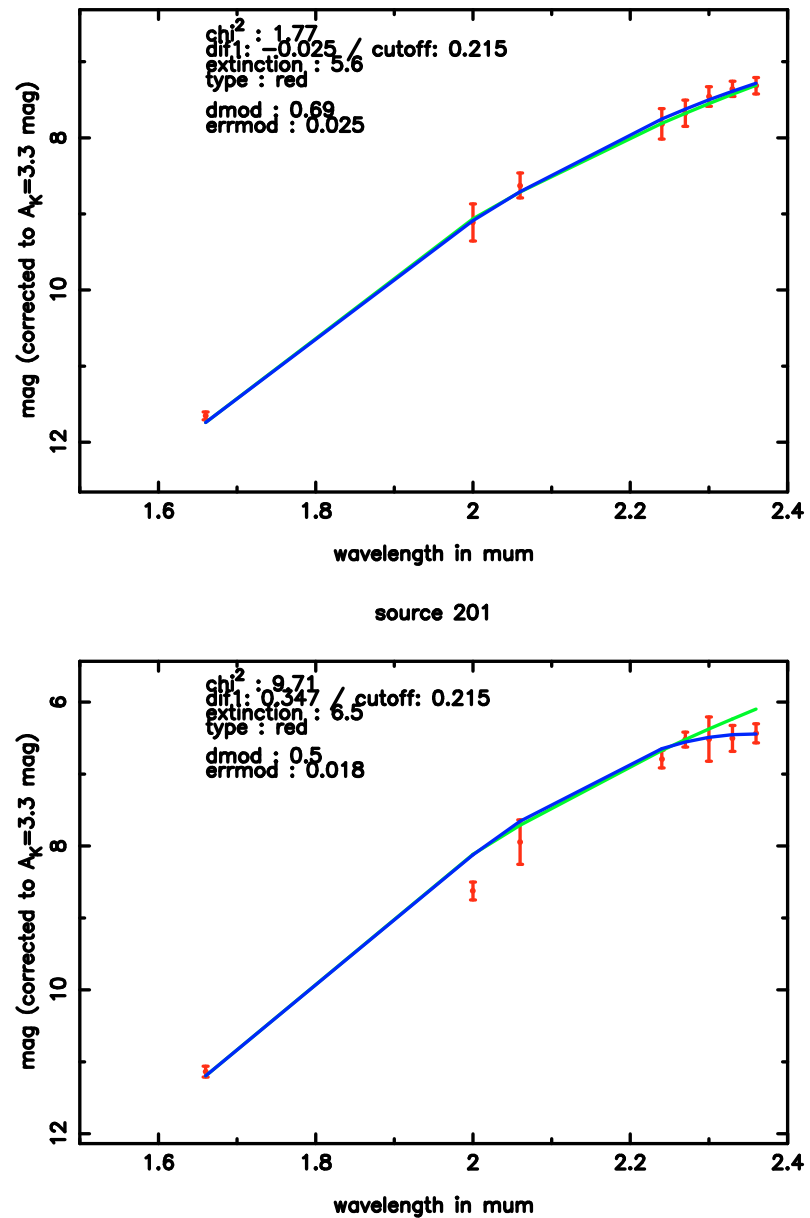

source 5908
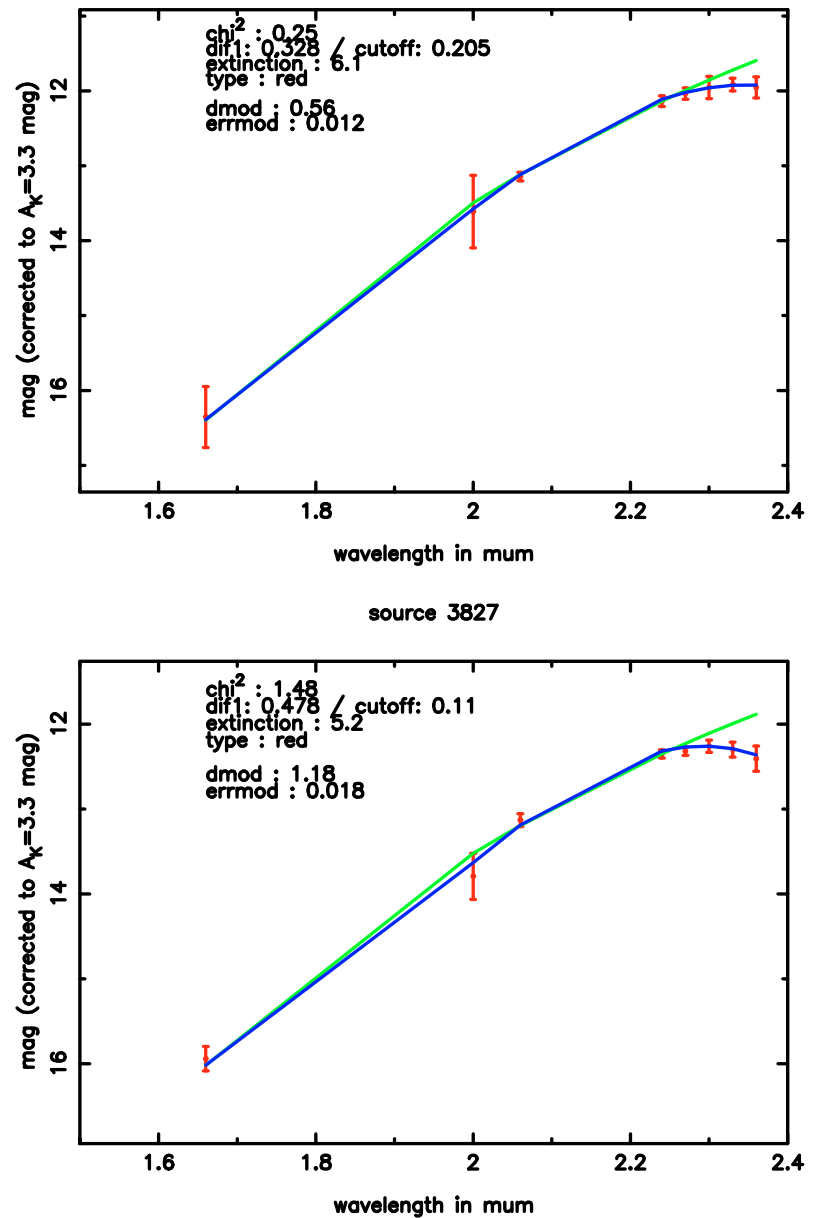

Fig. 18. SEDs of Extremely Red Objects. Upper left: source NW of Sgr A*, no CO feature, but strongly reddened. Upper right: source NW of Sgr A*, here a CO feature is visible. This source is located near a local maximum of extinction, so it may just be a normal late type star. Lower left: source located in the northern arm of the mini-spiral. Lower right: source located in the far SE of Sgr A*, also in a region of very high extinction. The CO feature suggest a late type star, maybe towards the back of the cluster.

These high rates of correct classifications gives us high confidence in our results, but the new detections need to be confirmed spectroscopically, especially the early type candidates.

2. The spatial distribution of the early type stars follows a power law, with $\beta_{1^{\prime \prime}}=1.49 \pm 0.12$, which is flatter than the values of the value of $\beta=-1.8$ we computed from the data published by Paumard et al. (2006), Lu et al. (2009), but still compatible at the $3 \sigma$ limit. It is also possible to fit the early type density with a broken power law with $\beta_{1-10^{\prime \prime}}=-1.08 \pm 0.12$ and $\beta_{10-20^{\prime \prime}}=-3.46 \pm 0.58$. This broken power law fits the density distribution better than the single power law, but it remains unknown what causes the density drop we observe at $\sim 10^{\prime \prime}$. The significance of this feature should be examined by further observations that extend to larger distances from Sgr A*.

3. We confirm the previously reported flat projected surface density profile of the late type stars in the innermost arcseconds with much higher source numbers and therefore significantly improved statistics. The flat (or even inversed) surface density of late type stars must imply a central dip or hole in their 3D distribution. This together with the steep early type density profile explains the observed drop in $\mathrm{CO}$ absorption. We would like to emphasize that this result signifies a change in our perception of the nuclear star cluster. Separating the early- and late-type population shows clearly that the GC cluster does not have a cusp (see discussions in Genzel et al. 2003; Schödel et al. 2007). To the contrary, the late-type, old stellar population, which appears to make up the vast majority of stars in the GC cluster, shows a flat or even slightly inverted power-law in projection within about $0.2 \mathrm{pc}$ from $\mathrm{Sgr} \mathrm{A}^{*}$. This means that there is some kind of hole in the late-type population near the center, as has been pointed out by Figer et al. (2003). The exact cause is still not understood, but various explanations have been suggested. One often discussed possibility is the destruction of the envelopes of giant stars by collisions with main-sequence stars, post-main sequence stars, and stellar remnants in the dense environment near Sgr A* (Rasio \& Shapiro 1990; Davies et al. 1991; Genzel et al. 1996; Alexander et al. 1999; Davies et al. 1998; Bailey \& Davies 1999; Dale et al. 2008). Another possible explanation for the non-existent cusp of late-type stars is that the cusp may have been destroyed by the infall of a second black hole (Merritt \& Szell 2006) and that there may not have been sufficient time yet to re-grow the cusp.

4. The late type KLF has a power law slope of $0.30 \pm 0.01$. This resembles closely the KLF that has been measured for the bulge of the Milky Way. This is surprising considering 
that the nuclear star cluster is probably a dynamically separate entity from the bulge (see Böker et al. 2008, for an overview of the properties of nuclear star clusters in galaxies). It implies a similar star formation history for the NSC and the bulge. The early type KLF has a much flatter slope of $(0.14 \pm 0.02)$. The fact that the flatter early type KLF could be confirmed down to our magnitude limit of $15.5 \mathrm{mag}$ is an important extension of previous works that had a completeness limit of 13-14 mag. Paumard et al. (2006) claimed that their flat early type KLF agreed best with stellar evolution models using a top-heavy initial mass function (IMF), but did not give a value for the slope of the KLF itself (Fig. 13 in that work). Our early type KLF seems to show a very similar shape down to our magnitude limit and thus also appears to support the proposed top-heavy IMF, but an accurate comparison is not possible due to the lack of a value for the slope in Paumard et al. (2006).

5. Early type stars have been detected outside of $0.5 \mathrm{pc}$, in a density that still agrees with the power law density profile in the inner region, so these sources could well be part of the known disk/off-disk population, if the single power law is valid. If the edge we observe at $\sim 10^{\prime \prime}$ is a real feature, this may point to a more complicated situation. This result is of course pending spectroscopic confirmation. We find a larger number of early type sources in the whole cluster with the density distribution following the same power law as in Paumard et al. (2006). This means that our power law still agrees with our early type density outside of $0.5 \mathrm{pc}$ that is higher than the upper limit provided in that work.

6. Both foreground stars and strongly reddened objects could be detected or excluded easily due to their fitted extinction. Unfortunately not all known bow-shock and mini-spiral sources could be observed, due to position and photometric uncertainties.

It is still not decided which one of the two main scenarios serves best to explain the presence of the early type stars in the central half parsec, in situ formation or infall and dissolution of a cluster formed at several parsecs distance from the GC. Recent publications lean towards favoring the scenario of in situ star formation (e.g. Paumard et al. 2006; Nayakshin \& Sunyaev 2005; Bartko et al. 2009). Our results also seem to agree best with this model: the steep power law decline of the projected early type density that agrees much better with the $R^{-2}$ power law expected for that scenario than with the $R^{-0.75}$ of the in-spiraling cluster. It is important to note that the cluster infall scenario predicts that less massive stars are stripped from the cluster at larger distances (Guerkan \& Rasio 2005). Our analysis probes, for the first time, the density of late-O/early B-type stars in the entire central parsec. These stars are less massive than the early-type stars reported from spectroscopic observations (Paumard et al. 2006; Bartko et al. 2009). Although we find a somewhat flatter powerlaw for the density of the early-type stars, the improved statistics still imply a steep decrease of the early-type stellar density with distance from Sgr A*. This supports the in situ scenario, but it is not possible to clearly rule out or confirm one scenario based on the current data.

Further observations that cover a larger area should be undertaken in the future, as well as spectroscopic confirmations of our new early type candidates. Lu et al. (2009) suggest covering at least the inner $5 \mathrm{pc}$ of the GC, and while observations of such an area require an extreme amount of time with an instrument like SINFONI, our method may be the key to explore large areas like this for features like tidal tails of an in-falling cluster or a continuation of the early type population of the central parsec.

Acknowledgements. We are grateful to all members of the NAOS/CONICA and the ESO PARANAL team. R. Schödel acknowledges support by the Ramón y Cajal programme by the Ministerio de Ciencia e Innovación of the government of Spain. We would also like to thank the referee for his helpful comments.

\section{References}

Alexander, T. 1999, ApJ, 527, 835

Alexander, T., \& Livio, M. 2004, ApJ, 606, L21

Alexander, T., \& Sternberg, A. 1999, ApJ, 520, 137

Allen, D. A., Hyland, A. R., \& Hillier, D. J. 1990, MNRAS, 244, 706

Bailey, V. C., \& Davies, M., B. 1999, MNRAS, 308, 257

Bartko, H., Martins, F., Fritz, T. K., et al. 2009, ApJ, accepted, [arXiv:0811.3903]

Becklin, E. E., Mathews, K., Neugebauer, G., \& Willner, S. P. 1978, ApJ, 219, 121

Blum, R. D., Sellgren, K., \& DePoy, D. L. 1996, AJ, 112, 1988

Blum, R. D., Ramírez, S. V., Sellgren, K., \& Olsen, K. 2003, ApJ, 597, 323

Böker, T., et al. 2008, Proceedings of The Universe Under the Microscope Astrophysics at High Angular Resolution, held 21-25 April 2008, in Bad Honnef, Germany, ed. R. Schödel, A. Eckart, S. Pfalzner, \& E. Ros, J. Phys. Conf. Ser., 131, 012043

Dale, J. E., Davies, M. B., Church, R. P., \& Freitag, M. 2008, MNRAS, accepted, [arXiv: 0811.3111]

Davidge, T. J., Simmons, D. A., Rigaut, F., Doyon, R., \& Crampton, D. 1997, AJ, 114, 2586

Davies, M. B., Benz, W., \& Hills, J. G. 1991, ApJ, 381, 449

Davies, M. B., Blackwell, R., Bailey, V. C., \& Sigurdsson, S. 1998, MNRAS, 301,745

Diolaiti, E., Bedinelli, O., Bonaccini, D., et al. G. 2000, A\&AS., 147, 335

Draine, B. T. 1989, in Interstellar extinction in the infrared, Infrared Spectroscopy in Astronomy, Proc. of the 22nd Eslab Symp. held in Salamanca, Spain, 7-9 December, 1988, ed. B. H. Kaldeich, ESA SP-290, 93

Eckart, A., Genzel, R., Hofmann, R., Sams, B. J., \& Tacconi-Garman, L. E. 1993, ApJ, 407, 77

Eckart, A., Ott, T., Genzel, R., \& Lutz, D. 1999, Proc. IAU Symp., 193, 449

Eckart, A., Genzel, R., Ott, T., \& Schödel, R. 2002, MNRAS, 331, 917

Eckart, A., Moultaka, J., Viehmann, T., Straubmeier, C., \& Mouawad, N. 2004, ApJ, 602, 760

Eisenhauer, F., Genzel, R., Alexander, T., et al. 2005, ApJ, 628, 246

Figer, D., Gilmore, D., Sungsoo, S., et al. 2003, ApJ, 599, 1139

Figer, D. F., Rich, R. M., Sungsoo, S. K., Morris, M., \& Serabyn, E. 2004, ApJ, 601,319

Freitag, M., Dale, J. E., Church, R. P., \& Davies, M. B. 2008, Proc. IAU Symp., 245,2007

Geballe, T. R., Rigaut, F., Roy, J.-R., \& Draine, B. T. 2004, ApJ, 602, 770

Geballe, T. R., Najarro, F., Rigaut, F., \& Roy, J.-R. 2006, ApJ, 652, 370

Genzel, R., Thatte, N., Krabbe, A., Kroker, H., \& Tacconi-Gharman, L. E., 1996, ApJ, 472, 153

Genzel, R., Schödel, R., Ott, T., et al. 2003, ApJ, 594, 812

Gerhard, O., 2001, ApJ, 546, L39

Ghez, A. M., Duchêne, G., Matthews, K., et al. 2003, ApJ, 586, L127

Ghez, A. M., Salim, S., Hornstein, S. D., et al. 2005, ApJ, 620, 744

Ghez, A. M., Salim, S., Weinberg, N. N., et al. 2008, ApJ, 689, 1044

Gillessen, S., Eisenhauer, F., Trippe, S., et al. 2009, ApJ, 692, 1075

Goodman, J. 2003, MNRAS, 339, 937

Groenewegen, M. A. T., Udalski, A., \& Bono, G. 2008, A\&A, 481, 441

Guerkan, M. A., \& Rasio, F. A. 2005, ApJ, 628, 236

Haller, J. W., Rieke, M. J., Rieke, G. H., et al. 1996, ApJ, 456, 194

Holland, S. 1998, AJ, 115, 1916

Kim, S. S., \& Morris, M. 2003, ApJ, 597, 312

Kim, S. S., Figer, D. F., \& Morris, M. 2004, ApJ, 607, L123

Krabbe, A., Genzel, R., Eckart, A., et al. 1995, ApJ, 447, L95

Lebofsky, M. J., \& Rieke, G. H. 1987, The Galactic Center, ed. D. C. Backer (New York: AIP), AIP Conf., 155, 79

Levin, Y., \& Beloborodov, A. M. 2003, ApJ, 590, L33

Lu, J. R., Ghez, A. M., Hornstein, S. D., Morris, M. R., \& Becklin, E. E. 2005, ApJ, 625, L51

Lu, J. R., Ghez, A. M., Hornstein, S. D., et al. 2009, ApJ, 690, 1463

Maillard, J. P., Paumard, T., Stolovy, S. R., \& Rigaut, F. 2004, A\&A, 423, 155

Maness, H., Martins, F., Trippe, S., et al. 2007, ApJ, 669, 1024

Martins, F., Genzel, R., Hillier, D. H., et al. 2007, A\&A, 468, 233 
McMillan, S. L. W. \& Portegies Zwart, S. F. 2003, ApJ, 596, 314

Merritt, D., \& Szell, A. 2006, ApJ, 648, 890

Milosavljevic, M., \& Loeb, A. 2004, ApJ, 604, L45

Morris, M. 1993, ApJ, 408, 496

Morris, P. W., Eenens, R. P. J., Hanson,. M. M., Conti, P. S., \& Blum, R. D. 1996, ApJ, 470, 597

Moultaka, J., Eckart, A., Schödel, R., Viehmann, T., \& Najarro, F. 2005, A\&A, 443,163

Muzic, K., Schödel, R., Eckart, A., Meyer, L., \& Zensus, A. 2008, A\&A, 482, 173

Najarro, F., Hillier, D. J., Kudritzki, R. P., et al. 1994, A\&A, 285, 573

Najarro, F., Krabbe, A., Genzel, R., et al. 1997, A\&A, 325, 700

Nayakshin, S., \& Cuadra, J. 2005, A\&A, 437, 437

Nayakshin, S., \& Sunyaev, R. 2005, MNRAS, 364, L23

Nishiyama, S., Nagata, T., Tamura, M., et al. 2008, ApJ, 680, 1174

Paumard, T., Maillard, J. P., Morris, M., \& Rigaut, F. 2001, A\&A, 366, 466

Paumard, T., Maillard, J.-P. \& Morris, M. 2004, A\&A, 426, 81

Paumard, T., Genzel, R., Martins, F., et al. 2006, ApJ, 643, 1011

Perger, M., Moultaka, J., Eckart, A., et al. 2008, A\&A, 478, 127
Portegies Zwart, S. F., McMillan, S. L. W., \& Gerhard, O. 2003, ApJ, 593, 352 Pott, J.-U., Eckart, A., \& Glindemann, A. 2008, A\&A, 480, 115

Rasio, F. A., \& Shapiro, S. L. 1990, ApJ, 354, 201

Reid, M. J. 1993, ARA\&A, 31, 345

Rieke, G. H., Rieke, M. J., \& Paul, A. E. 1989, ApJ, 336, 752

Schödel, R., Ott, T., Genzel, R., et al. 2002, Nature, 419, 694

Schödel, R., Ott, T., Genzel, R., et al. 2003, ApJ, 596, 1015

Schödel, R., Eckart, A., Alexander, T., et al. 2007, A\&A, 469, 125

Schödel, R., Eckart, A., Buchholz, R., et al., in preparation

Scoville, N. Z., Stolovy, S. R., Rieke, M., Christopher, M., \& Yusef-Zadeh, F. 2003, ApJ, 594, 294

Sellgren, K, McGinn, M. T., Becklin, E. E., \& Hall, D. N. 1990, ApJ, 359, 112

Tanner, A., Ghez, A. M., Morris, M., et al. 2002, ApJ, 575, 860

Tanner, A., Ghez, A. M., Morris, M., \& Becklin, E. E. 2003, ASN, 597

Tiede, G. P., Frogel, J. A., \& Terndrup, D. M. 1995, AJ, 110, 2788

Trippe, S., Gillessen, S., Gerhard, O. E., et al. 2008, A\&A, 492, 419

Wallace, L., \& Hinkle, K. 1997, ApJS, 111, 445

Zoccali, M., Renzini, A., Ortolani, S. et al. 2003, A\&A, 399, 931

Zhu, Q., Kudritzki, R., Figer, D., Najarro, F., \& Merritt, D. 2008, ApJ, 681, 1254 